\title{
Core concepts for incomplete market economies
}

Citation for published version (APA):

Habis, H., \& Herings, P. J. J. (2011). Core concepts for incomplete market economies. Journal of Mathematical Economics, 47(4-5), 595-609. https://doi.org/10.1016/j.jmateco.2011.07.005

Document status and date:

Published: 01/01/2011

DOI:

10.1016/j.jmateco.2011.07.005

Document Version:

Publisher's PDF, also known as Version of record

Document license:

Taverne

Please check the document version of this publication:

- A submitted manuscript is the version of the article upon submission and before peer-review. There can be important differences between the submitted version and the official published version of record.

People interested in the research are advised to contact the author for the final version of the publication, or visit the DOI to the publisher's website.

- The final author version and the galley proof are versions of the publication after peer review.

- The final published version features the final layout of the paper including the volume, issue and page numbers.

Link to publication

\footnotetext{
General rights rights.

- You may freely distribute the URL identifying the publication in the public portal. please follow below link for the End User Agreement:

www.umlib.nl/taverne-license

Take down policy

If you believe that this document breaches copyright please contact us at:

repository@maastrichtuniversity.nl

providing details and we will investigate your claim.
}

Copyright and moral rights for the publications made accessible in the public portal are retained by the authors and/or other copyright owners and it is a condition of accessing publications that users recognise and abide by the legal requirements associated with these

- Users may download and print one copy of any publication from the public portal for the purpose of private study or research.

- You may not further distribute the material or use it for any profit-making activity or commercial gain

If the publication is distributed under the terms of Article $25 \mathrm{fa}$ of the Dutch Copyright Act, indicated by the "Taverne" license above, 


\title{
Core concepts for incomplete market economies
}

\author{
Helga Habis ${ }^{\mathrm{a}, \mathrm{b}}$, P. Jean-Jacques Herings ${ }^{\mathrm{c}, *}$ \\ ${ }^{a}$ Institute of Economics, Hungarian Academy of Sciences, Hungary \\ b Department of Microeconomics, Corvinus University of Budapest, Fővám tér 8., 1093, Budapest, Hungary \\ ${ }^{\mathrm{c}}$ Department of Economics, Universiteit Maastricht, P.O. Box 616, 6200 MD, Maastricht, The Netherlands
}

\section{A R T I C L E I N F O}

\section{Article history:}

Received 4 June 2010

Received in revised form

7 June 2011

Accepted 26 July 2011

Available online 19 August 2011

\section{Keywords:}

Incomplete markets

Dynamic core concepts

Time and uncertainty

\section{Introduction}

We examine the notion of the core in the standard two-period general equilibrium model with incomplete markets. Market incompleteness implies that players cannot make all possible binding commitments regarding their actions at different date-events. In the literature, a number of proposals can be found for the appropriate notion of the core in a context with restricted commitment possibilities. Many of these contributions were developed independently, and in environments as distinct as economies with incomplete markets, economies with transaction costs, dynamic monetary economies, deterministic capital accumulation models, and sequences of transferable utility games.

We unify the various treatments of dynamic core concepts that so far are scattered around in the literature, and find that several of the concepts proposed actually coincide. This results in definitions of the Classical Core, the Segregated Core (Grossman, 1977; Bester, 1984; Repullo, 1988a), the Two-stage Core (Koutsougeras, 1998), the Strong Sequential Core (Gale, 1978; Becker and Chakrabarti, 1995; Predtetchinski et al., 2002; Kranich et al., 2005), and the Weak Sequential Core (Kranich et al., 2005; Predtetchinski et al., 2006). Except for the Classical Core, all these concepts can be

\footnotetext{
* Corresponding author.

E-mail addresses: helga.habis@uni-corvinus.hu (H. Habis), P.Herings@algec.unimaas.nl (P.J.J. Herings).
}

defined by requiring the absence of blocking in period 0 and at any date-event in period 1 . The concepts only differ with respect to the notion of blocking in period 0 .

Consider a particular allocation and portfolio plan. Since the only commitment possibilities are those implied by the portfolio plan, a coalition can block at a date-event in period 1 if it can redistribute its initial endowments and proceeds from the portfolio plan in such a way as to make every coalition member better off. All the core concepts, with the exception of the Classical Core, agree with this notion of blocking. The Classical Core is essentially a static concept and ignores the option of blocking at a date-event in period 1.

To assess whether a coalition blocks in period 0 , it has to evaluate the consequences of a deviation regarding consumption in period 1. It is here that the various concepts differ. In the Segregated Core, it is assumed that net trades in period 1 are not affected by a deviation in period 0 . The Two-stage Core takes a very conservative point of view in that coalition members are only guaranteed their initial endowments plus the proceeds from their asset portfolio. The Strong Sequential Core agrees with the Classical Core in that, it regards any future redistribution of endowments as feasible. Since, contrary to the Classical Core, the Strong Sequential Core allows for blocking in period 1 , it is a refinement of the Classical Core. For the Weak Sequential Core, it is assumed that coalition members can coordinate on a particular element of the core of the ex-post economies in period 1 that result after a deviation. 
We evaluate these core concepts for three different market structures: strongly complete markets, incomplete markets in finance economies, and incomplete markets in settings with multiple commodities. Markets are said to be strongly complete if every consumption bundle can be implemented today by means of the existing assets. Finance economies are economies in which contingent on each date-event, there is exactly one commodity being traded. For finance economies we do not impose assumptions on the market structure. Finally, we study the multiple commodity case with a general market structure.

One may expect that when markets are strongly complete all core concepts coincide. However, such is not the case. The only two concepts that coincide are the Two-stage Core and the Strong Sequential Core. Both cores are contained in the Weak Sequential Core and the Classical Core, but there is no general relationship between the latter two. The Segregated Core does not satisfy any general relationship with any of the other concepts. We argue that the Classical Core is not restrictive enough for dynamic economies with strongly complete markets, as it does not take into account new blocking opportunities that arise in the future. The Classical Core is therefore not an appropriate concept to study dynamic economies. The Segregated Core on the other hand is too permissive, as it may even include allocations that fail to be individually rational, which also discards the Segregated Core as a reasonable concept. When we impose some additional assumptions, in particular the assumption that the Classical Core of relevant ex-post economies is non-empty and the assumption that Strong and Weak Pareto Optimal allocations coincide, we can show that all core concepts coincide with the exception of the Segregated Core, which is shown to contain the other concepts.

In finance economies, i.e. economies where one commodity per date-event is being traded, and a general market structure, it is still true that the Two-stage Core and the Weak Sequential Core coincide, and for finance economies these two concepts even coincide with the Segregated Core. The equivalence with the Classical Core and the Strong Sequential Core is now lost, due to the potential market incompleteness. The Strong Sequential Core is a proper subset of all the other concepts, whereas apart from the relation to the Strong Sequential Core, the Classical Core does not satisfy other relationships. In the extreme case of finance economies without asset markets, the Strong Sequential Core is typically empty, the Classical Core includes some Pareto efficient allocation, and the other concepts coincide with the initial endowments, the only reasonable prediction in this case. It follows that the Strong Sequential Core is not an appropriate concept when studying economies with incomplete markets.

In the general case - multiple commodities and potentially incomplete asset markets - we show that competitive equilibria belong to the Segregated Core and the Two-stage Core. In general it is not true that competitive equilibria belong to the Classical Core, the Strong Sequential Core, and the Weak Sequential Core. This is an indication that the Segregated Core and the Twostage Core are too permissive. The constrained suboptimality results of Geanakoplos and Polemarchakis (1986) state that competitive equilibria are not constrained optimal, so can typically be improved upon while only making use of the existing assets in the economy. It is then only natural that competitive equilibria typically do not belong to an appropriate concept of a dynamic core. We are left with the Weak Sequential Core as the only concept that does not suffer from major deficiencies. We show that in the general case, the Strong Sequential Core is a subset of the Classical Core and the Weak Sequential Core, and that the Weak Sequential Core is a subset of the Two-stage Core. Examples illustrate that there are no further relationships.

The outline of the paper is as follows. We specify the model in Section 2 and give the formal definitions of the various core concepts in Section 3. We compare these concepts for the case with strongly complete markets in Section 4 . The one-commodity case is studied in Section 5. Section 6 examines the relation of the core concepts and the competitive equilibrium. We discuss the general case with incomplete markets and multiple commodities in Section 7. Section 8 concludes.

\section{The model}

Consider an economy with two time-periods, $t \in\{0,1\}$. In time-period 1 trade takes place conditional on the occurrence of a date-event $s$ in the finite set of date-events $S$. We define the dateevent for time-period 0 as $s=0$, so the set of all date-events is $S^{\prime}=\{0\} \cup S$. At each date-event there is trade in a finite set $L$ of non-durable consumption goods.

There is a finite number of households $h \in H$ who participate in the economy. Household $h$ has initial endowments $e^{h}=$ $\left(e_{s}^{h}\right)_{s \in S^{\prime}} \in \mathbb{R}^{S^{\prime} L}$. The profile of initial endowments is $e=\left(e^{h}\right)_{h \in H}$. The preferences of household $h$ are represented by its utility function $u^{h}: X^{h} \rightarrow \mathbb{R}$, with the consumption set $X^{h}$ a subset of the commodity space $\mathbb{R}^{S^{\prime} L}$. We denote $\prod_{h \in H} X^{h}$ by $X$, with typical element $x$. Let $C$ be the collection of all coalitions, i.e. the collection of all non-empty subsets of $H$. For $C \in \mathcal{C}$, we denote $\prod_{h \in C} X^{h}$ by $X^{C}$, with typical element $x^{C}$.

For $\bar{s} \in S^{\prime}$, we denote the consumption $\left(x_{s}^{h}\right)_{s \in S^{\prime} \backslash\{\bar{s}\}}$ of a household $h$ outside date-event $\bar{s}$ by $x_{-\bar{s}}^{h}$. The utility function $u^{h}$ is locally nonsatiated in date-event $\bar{s} \in S^{\prime}$ if for every $\bar{x}^{h} \in X^{h}$ and for every $\varepsilon>0$ there is $x^{h} \in X^{h}$ with $x_{-\bar{s}}^{h}=\bar{x}_{-\bar{s}}^{h}$ such that $\left\|x_{\bar{s}}^{h}-\bar{x}_{\bar{s}}^{h}\right\|_{\infty}<\varepsilon$ and $u^{h}\left(x^{h}\right)>u^{h}\left(\bar{x}^{h}\right)$.

For $\bar{x}_{0}^{h} \in \mathbb{R}^{L}$ we define the set $X^{h}\left(\bar{x}_{0}^{h}\right)=\left\{x^{h} \in X^{h} \mid x_{0}^{h}=\bar{x}_{0}^{h}\right\}$ as the set of feasible consumption bundles with state 0 consumption equal to $\bar{x}_{0}^{h}$. The consumption set $X^{h}$ is said to be state separable if for every $x_{0}^{h} \in \mathbb{R}^{L}$ the set $X^{h}\left(x_{0}^{h}\right)$ is either empty or it has the product form $\left\{x_{0}^{h}\right\} \times \prod_{s \in S} X_{s}^{h}\left(x_{0}^{h}\right)$, where $X_{s}^{h}\left(x_{0}^{h}\right)$ is a subset of $\mathbb{R}^{L}$. For state separable $X^{h}$, we define the set

$X_{0, s}^{h}=\cup_{x_{0}^{h} \in \mathbb{R}^{L}}\left\{x_{0}^{h}\right\} \times X_{s}^{h}\left(x_{0}^{h}\right), \quad s \in S$,

with the convention that a product involving an empty set is empty itself. The utility function $u^{h}$ is said to be state separable if there exist functions $u_{s}^{h}: X_{0, s}^{h} \rightarrow \mathbb{R}, s \in S$, such that $u^{h}\left(x^{h}\right)=$ $\sum_{s \in S} u_{s}^{h}\left(x_{0}^{h}, x_{s}^{h}\right)$.

We apply the following assumption throughout the paper.

Assumption 2.1. For $h \in H, X^{h}$ is non-empty, closed, convex, and state separable, and the utility function is continuous, state separable, and locally non-satiated in every date-event. ${ }^{1}$

Von Neumann-Morgenstern utility functions would be a prominent example of utility functions satisfying Assumption 2.1. State separability is a natural requirement since only one out of the future states of nature materializes.

At date 0 , there is a finite set $J$ of assets. An asset $j \in J$ pays a dividend $d_{s j} \in \mathbb{R}^{L}$ at date-event $s \in S$. We denote the $(L \times J)$ matrix of dividends by $D_{s}=\left(d_{s j}\right)_{j \in J}$ and the $(S L \times J)$-asset payoff matrix by $A=\left(D_{s}\right)_{s \in S}$. We assume that assets are in zero net supply. At date-event 0 , household $h$ chooses a portfolio holding $\theta^{h} \in \mathbb{R}^{J}$ and a consumption bundle $x_{0}^{h} \in \mathbb{R}^{L}$. Households choose a consumption bundle $x_{s}^{h}$ conditional on $s$ at date-events in $S$. The only commitments households can make regarding the future are those implied by their portfolio holding $\theta^{h}$. We denote $\prod_{h \in H} \mathbb{R}^{J}$ by

\footnotetext{
1 Most of our results do not rely on $X^{h}$ being non-empty, closed, and convex. We merely make these assumptions to rule out pathological cases.
} 
$\Theta$, with typical element $\theta$, and, for $C \in \mathcal{C}, \prod_{h \in C} \mathbb{R}^{J}$ by $\Theta^{C}$, with typical element $\theta^{C}$. As it is standard in the incomplete markets literature, we focus attention on the case without constraints on portfolio holdings. An interesting extension for future research is to allow $\Theta^{\{i\}}$ to be a proper subset of $\mathbb{R}^{J}$.

The economy $\varepsilon=\left(\left(X^{h}, e^{h}, u^{h}\right)_{h \in H}, A\right)$ is defined by the households' consumption sets, initial endowments, utility functions, and the asset payoff matrix.

\section{Core concepts}

\subsection{Attainability and ex post feasibility}

In this section we study which allocations $x \in X$ and portfolio plans $\theta \in \Theta$ are stable in an economy $\mathcal{E}$. In general, $(\bar{x}, \bar{\theta}) \in X \times \Theta$ is stable if there is no date-event $s \in S^{\prime}$ and no coalition $C$ that can improve upon $(\bar{x}, \bar{\theta})$ at date-event $s$, i.e. there does not exist $s \in S^{\prime}$ and $\left(x^{C}, \theta^{C}\right) \in X^{C} \times \Theta^{C}$ that is feasible for coalition $C$ at $s$ which yields higher utility than $(\bar{x}, \bar{\theta})$ for each member of $C$.

The general definition of the previous paragraph reduces the question of stability to the question of feasibility for a coalition at a date-event. We reformulate the definitions of feasibility that so far are scattered around in the literature and have been considered for different environments. We apply them to economies $\varepsilon$ as defined in Section 2. This results in five definitions: the Classical Core CC $(\varepsilon)$, the Segregated Core SC $(\varepsilon)$, the Two-stage Core TSC $(\varepsilon)$, the Strong Sequential Core $\operatorname{SSC}(\mathscr{E})$, and the Weak Sequential Core WSC $(\mathscr{E})$. We devote one subsection to each particular definition. We illustrate the five concepts with a simple example with $L=\{1\}$ and $J=\emptyset$.

In the following subsections we first present the Classical Core, followed by the truly dynamic core concepts. The definitions that we give follow a common structure. First we define the feasibility for a coalition $C$ at a date-event $s \in S$, referred to as ex post feasibility, next feasibility for a coalition $C$ at date-event 0 , then the notion of improvement, and finally the core concept itself. Some basic relationships between these core concepts are summarized in Section 3.8.

First, we define attainability, a concept weaker than feasibility, and only specifying that accounting should be done correctly.

Definition 3.1.1. An allocation and portfolio plan $(\bar{x}, \bar{\theta}) \in X \times \Theta$ is attainable in the economy $\mathbb{E}$ if

$\sum_{h \in H} \bar{x}^{h}=\sum_{h \in H} e^{h}$,
$\sum_{h \in H} \bar{\theta}^{h}=0$.

All dynamic core concepts studied in this paper will coincide for date-events in period 1 . These core concepts result in the Classical Core for an economy with one time-period only and initial endowments given by the original initial endowments plus the dividends yielded by the asset portfolio conditional on the dateevent reached. This motivates the following definition of ex post feasibility.

Definition 3.1.2. Let some allocation and portfolio plan $(\bar{x}, \bar{\theta}) \in$ $X \times \Theta$ be given. Then $\left(x^{C}, \theta^{C}\right) \in X^{C} \times \Theta^{C}$ is ex post feasible for coalition $C \in \mathcal{C}$ at date-event $\bar{s} \in S$ if

$x_{-\bar{s}}^{h}=\bar{x}_{-\bar{s}}^{h}, \quad h \in C$,

$\theta^{h}=\bar{\theta}^{h}, \quad h \in C$,

$\sum_{h \in C} x_{\bar{s}}^{h}=\sum_{h \in C}\left(e_{\bar{s}}^{h}+D_{\bar{s}} \theta^{h}\right)$.

Definition 3.1.2 expresses the ex post feasible allocations and portfolio plans for a coalition $C$ at date-event $\bar{s} \in S$ given some allocation and portfolio plan $(\bar{x}, \bar{\theta})$. The first two conditions require that the members of a coalition take consumption bundles outside state $\bar{s}$ and portfolio holdings as given. The last equality in the definition states that, following the resolution of uncertainty at date-event $\bar{s}$, executed asset contracts serve as initial endowments which can be redistributed among the members of the coalition. This definition therefore incorporates that the only binding commitments regarding the future are those implied by the portfolio holdings $\bar{\theta}$. Given some $(x, \theta) \in X \times \Theta$, we refer to $\left(x_{0}^{h},\left(e_{-0}^{h}+A \theta^{h}\right)\right)$ as the intermediate consumption bundle and to $\left(x_{0}^{h},\left(e_{-0}^{h}+A \theta^{h}\right)\right)_{h \in H}$ as the intermediate allocation.

\subsection{The Classical Core $\mathrm{CC}(\mathcal{E})$}

The Classical Core implicitly assumes that all commitments regarding the future are binding. As such it is not an appropriate concept to define stability in our set-up. We will argue that this is even the case when asset markets are strongly complete. The following sequence of definitions is entirely standard.

Definition 3.2.1. The allocation $x^{C} \in X^{C}$ is CC-feasible for a coalition $C \in \mathcal{C}$ if

$\sum_{h \in C} x^{h}=\sum_{h \in C} e^{h}$.

Definition 3.2.2. Let some allocation $\bar{x} \in X$ be given. A coalition $C \in \mathcal{C}$ can CC-improve upon $\bar{x}$ if there exists a CC-feasible allocation $x^{C} \in X^{C}$ for $C$ such that

$u^{h}\left(x^{h}\right)>u^{h}\left(\bar{x}^{h}\right), \quad h \in C$.

Definition 3.2.3. The Classical Core of the economy $\mathcal{E}$, denoted by CC $(\mathcal{E})$, is the collection of allocations $\bar{x} \in X$ such that $\sum_{h \in H} \bar{x}^{h}=$ $\sum_{h \in H_{-}} e^{h}$, and there is no coalition $C \in \mathcal{C}$ that can CC-improve upon $\bar{x}$.

The Classical Core is non-empty when consumption sets are bounded from below, standard quasi-concavity assumptions are imposed on the utility functions, and initial endowments are assumed to belong to consumption sets. Allocations in the Classical Core are individually rational and weakly Pareto efficient.

Now we turn to the truly dynamic core concepts.

\subsection{The Segregated Core $\mathrm{SC}(\mathcal{E})$}

This subsection reformulates three concepts that appeared before in the literature under different names: the Social Nash Optimum (Grossman, 1977), the Core (Bester, 1984), and the Segregated Core (Repullo, 1988a). These concepts are essentially the same, though originally they were defined in different settings. The Social Nash optimum was not formulated for coalitions, but only used as an optimality criterion. It was later generalized by Repullo (1988b) as D-efficiency. In Bester (1984) there are infinitely many households, represented by the unit interval, and in Repullo (1988a) there are no securities, but transaction technologies that are costly to carry out. We follow Repullo (1988a) and call this concept the Segregated Core.

Definition 3.3.1. Let some allocation and portfolio plan $(\bar{x}, \bar{\theta}) \in$ $X \times \Theta$ be given. Then $\left(x^{C}, \theta^{C}\right) \in X^{C} \times \Theta^{C}$ is SC-feasible for coalition $C \in \mathcal{C}$ at date-event 0 if

$x_{s}^{h}-\bar{x}_{s}^{h}=D_{s}\left(\theta^{h}-\bar{\theta}^{h}\right), \quad s \in S, h \in C$,

$\sum_{h \in C} x_{0}^{h}=\sum_{h \in C} e_{0}^{h}$,

$\sum_{h \in C} \theta^{h}=0$.

Definition 3.3.1 specifies the allocation that results from a deviation by coalition $C$ at date-event 0 . The coalition members can 
rearrange their date- 0 consumption and portfolio holdings, and when doing so, they expect the same net trades to take place in period 1.

Definition 3.3.2. Let some allocation and portfolio plan $(\bar{x}, \bar{\theta}) \in$ $X \times \Theta$ be given. A coalition $C \in \mathcal{C}$ can SC-improve upon $(\bar{x}, \bar{\theta})$ at date-event $\bar{s} \in S^{\prime}$ if there exists an SC-feasible $\left(x^{C}, \theta^{C}\right) \in X^{C} \times \Theta^{C}$ for $C$ at $\bar{s}=0$ or an ex post feasible $\left(x^{C}, \theta^{C}\right) \in X^{C} \times \Theta^{C}$ for $C$ at $\bar{s} \in S$ such that

$u^{h}\left(x^{h}\right)>u^{h}\left(\bar{x}^{h}\right), \quad h \in C$.

Throughout the paper we will say that a coalition can block at a particular date-event if it has some improvement at that dateevent.

Definition 3.3.3. The Segregated Core of the economy $\varepsilon$, denoted by $\operatorname{SC}(\varepsilon)$, is the collection of attainable allocations and portfolio plans $(\bar{x}, \bar{\theta}) \in X \times \Theta$ such that there is no date-event $\bar{s} \in S^{\prime}$ at which some coalition $C \in \mathcal{C}$ can SC-improve upon $(\bar{x}, \bar{\theta})$.

We now illustrate this concept for an economy with $L=\{1\}$ and $J=\emptyset$.

Example 3.3.4. Consider an economy $\varepsilon$ with increasing utility functions, $L=\{1\}$, and $J=\varnothing$. Applying the definitions, one can show first that there is no re-distribution at any date-event in time-period one, and making use of this fact that there is no redistribution in time-period zero, $\operatorname{so~} \mathrm{SC}(\mathscr{E})=\{e\}$ obtains.

The Classical Core is in general strikingly different from $\mathrm{SC}(\mathscr{E})$. The reason is obvious. The Classical Core assumes that all attainable allocations are enforceable. It is therefore equal to a set of particular weakly Pareto optimal allocations. The Segregated Core on the contrary specifies that only the no-trade allocation is stable if there are no commitments at all regarding the future.

\subsection{The Two-stage Core $\operatorname{TSC}(\varepsilon)$}

In this section, we first reformulate the Two-stage Core as introduced in Koutsougeras (1998) to allow for date-zero consumption, then we apply it to our example.

Definition 3.4.1. The allocation and portfolio plan $\left(x^{C}, \theta^{C}\right) \in X^{C} \times$ $\Theta^{C}$ is TSC-feasible for coalition $C \in \mathcal{C}$ at date-event 0 if

$x_{s}^{h}=e_{s}^{h}+D_{s} \theta^{h}, \quad s \in S, h \in C$,

$\sum_{h \in C} x_{0}^{h}=\sum_{h \in C} e_{0}^{h}$

$\sum_{h \in C} \theta^{h}=0$.

Definition 3.4.1 takes the completely conservative viewpoint that members of a deviating coalition at date-event 0 cannot engage in any further trade in the following period; they just consume the sum of their initial endowments and the payoff of their asset portfolio.

Definition 3.4.2. Let some allocation and portfolio plan $(\bar{x}, \bar{\theta}) \in$ $X \times \Theta$ be given. A coalition $C \in \mathcal{C}$ can TSC-improve upon $(\bar{x}, \bar{\theta})$ at date-event $\bar{s} \in S^{\prime}$ if there exists a TSC-feasible $\left(x^{C}, \theta^{C}\right) \in X^{C} \times \Theta^{C}$ for $C$ at $\bar{s}=0$ or an ex post feasible $\left(x^{C}, \theta^{C}\right) \in X^{C} \times \Theta^{C}$ for $C$ at $\bar{s} \in S$ such that

$u^{h}\left(x^{h}\right)>u^{h}\left(\bar{x}^{h}\right), \quad h \in C$.

Definition 3.4.3. The Two-stage Core of the economy $\mathcal{E}$, denoted by $\operatorname{TSC}(\mathcal{E})$, is the collection of attainable allocations and portfolio plans $(\bar{x}, \bar{\theta}) \in X \times \Theta$ such that there is no date-event $\bar{s} \in S^{\prime}$ at which some coalition $C \in \mathcal{C}$ can TSC-improve upon $(\bar{x}, \bar{\theta})$.
Example 3.3.4 (Continued). It is easy to verify that $\operatorname{TSC}(\mathscr{E})=$ $\{e\}$.

\subsection{The Strong Sequential Core $\operatorname{SSC}(\&)$}

In this section we reformulate the definition of the Strong Sequential Core as given by Predtetchinski et al. (2002). Note that the papers by Gale (1978), Becker and Chakrabarti (1995), and Kranich et al. (2005) present essentially the same core concept for the cases of a dynamic monetary economy, a deterministic capital accumulation model, and a deterministic sequence of TU-games, respectively. The latter three papers do not incorporate the set-up of this paper with a general set of asset markets.

Definition 3.5.1. The allocation and portfolio plan $\left(x^{C}, \theta^{C}\right) \in X^{C} \times$ $\Theta^{C}$ is SSC-feasible for coalition $C \in \mathcal{C}$ at date-event 0 if

$\sum_{h \in C} x^{h}=\sum_{h \in C} e^{h}$,
$\sum_{h \in C} \theta^{h}=0$.

Definition 3.5.2. Let some allocation and portfolio plan $(\bar{x}, \bar{\theta}) \in$ $X \times \Theta$ be given. A coalition $C \in \mathcal{C}$ can SSC-improve upon $(\bar{x}, \bar{\theta})$ at date-event $\bar{s} \in S^{\prime}$ if there exists an SSC-feasible $\left(x^{C}, \theta^{C}\right) \in X^{C} \times \Theta^{C}$ for $C$ at $\bar{s}=0$ or an ex post feasible $\left(x^{C}, \theta^{C}\right) \in X^{C} \times \Theta^{C}$ for $C$ at $\bar{s} \in S$ such that

$u^{h}\left(x^{h}\right)>u^{h}\left(\bar{x}^{h}\right), \quad h \in C$.

Definition 3.5.3. The Strong Sequential Core of the economy $\&$, denoted by $\operatorname{SSC}(\varepsilon)$, is the collection of attainable allocations and portfolio plans $(\bar{x}, \bar{\theta}) \in X \times \Theta$ such that there is no date-event $\bar{s} \in S^{\prime}$ at which some coalition $C \in \mathcal{C}$ can SSC-improve upon $(\bar{x}, \bar{\theta})$.

Contrary to the previous definitions, a coalition may redistribute the future resources of the coalition in any way. The Strong Sequential Core is therefore a refinement of the Classical Core.

There are a number of differences between our definition of $\operatorname{SSC}(\mathcal{E})$ and the definition of the Strong Sequential Core as given in Predtetchinski et al. (2002). In the latter paper the Strong Sequential Core is defined as the set of Classical Core allocations $\bar{x}$ of the economy $\mathcal{E}$ for which there is a feasible intermediate allocation such that, for every date-event $s \in S, \bar{x}_{s}$ belongs to the Classical Core of the corresponding ex-post economy. ${ }^{2}$ Rather than identifying what is feasible for each coalition at each date-event, and requiring the absence of improvements by any coalition at any date-event, Predtetchinski et al. (2002) therefore gives a more reduced definition of the Strong Sequential Core. Minor technical differences are that Predtetchinski et al. (2002) define the Strong Sequential Core as a subset of $X$ rather than $X \times \Theta$ and do not allow for consumption in period 0 . A more substantial difference is that they require the intermediate allocation to belong to $X$, whereas no such requirement is embodied in Definition 3.1.2. Our definition therefore corresponds to what Predtetchinski et al. (2002) call the Semi-strong Sequential Core, where it is not required that the intermediate consumption bundles be feasible. Since nothing prevents agents from holding non-feasible intermediate consumption bundles with the objective of future retrading in mind, we find this definition more compelling. Typical real life examples of non-feasible intermediate consumption

\footnotetext{
2 We refer the reader to Definition 3.6.1 for a formal treatment of an ex-post economy.
} 
bundles occur for instance when households buy a house and take a mortgage that is redeemed out of future labor income or firms finance an investment by a loan rather than by issuing equity.

However, the strong sequential core is too demanding, since it is empty for economically interesting cases, as is shown next.

Example 3.3.4 (Continued). We apply the concept of the Strong Sequential Core to our example. As before, it follows from Definition 3.1.2 that $\bar{x}_{s}^{h}=e_{s}^{h}$ for all $h \in H$ and $s \in S$ when $\bar{x}$ belongs to $\operatorname{SSC}(\mathcal{E})$. The conditions imposed by Definition 3.5.1 are the same as those of the Classical Core and imply individual rationality. Hence, $\bar{x} \in \operatorname{SSC}(\&)$ implies $\bar{x}_{s}^{h}=e_{s}^{h}$, for every $s \in S^{\prime}$, and $\bar{x} \in \mathrm{CC}(\mathcal{E})$. We find that

$\operatorname{SSC}(\varepsilon)=\emptyset, \quad$ if $e \notin \operatorname{CC}(\varepsilon)$,

$\operatorname{SSC}(\varepsilon)=\{e\}, \quad$ otherwise.

Since $e$ is typically not weakly Pareto efficient, we find that typically $\operatorname{SSC}(\varepsilon)=\emptyset$.

\subsection{The Weak Sequential Core $\operatorname{WSC}(\varepsilon)$}

In this section we reformulate the concept of the Weak Sequential Core as given by Predtetchinski et al. (2006). The idea of the Weak Sequential Core is already hinted at in Gale (1978).

Before introducing feasibility at date-event 0 , we introduce the notion of an ex-post economy for coalition $C$. The ex-post economy for coalition $C$ at a date-event in $S$ corresponds to an economy consisting of households in $C$, immediately after the realization of the date-event and the payment of the dividends.

Definition 3.6.1. Let some allocation and portfolio plan $(\bar{x}, \bar{\theta}) \in$ $X \times \Theta$ be given. The ex-post economy for coalition $C \in \mathcal{C}$ at dateevent $s \in S$ is defined by

$\mathcal{E}_{s, \bar{x}^{C}, \bar{\theta}^{c}}=\left(X_{s}^{h}, \bar{e}_{s}^{h}, u_{\mid X_{s}^{h}}^{h}\right)_{h \in C}$

where

$X_{s}^{h}=\left\{x^{h} \in X^{h} \mid x_{-s}^{h}=\bar{x}_{-s}^{h}\right\}$,

$\bar{e}_{s, s}^{h}=e_{s}^{h}+D_{s} \bar{\theta}^{h}$,

$\bar{e}_{s,-s}^{h}=\bar{x}_{-s}^{h}$.

Here we use the notation $u_{\mid X_{s}^{h}}^{h}$ for the restriction of the utility function $u^{h}$ to the consumption set $X_{s}^{h}$.

Definition 3.6.2. The allocation and portfolio plan $\left(x^{C}, \theta^{C}\right) \in X^{C} \times$ $\Theta^{C}$ is WSC-feasible for coalition $C \in \mathcal{C}$ at date-event 0 if

$x^{C} \in \mathrm{CC}\left(\mathcal{E}_{s, x^{C}, \theta^{C}}\right), \quad s \in S$,

$\sum_{h \in C} x_{0}^{h}=\sum_{h \in C} e_{0}^{h}$,

$\sum_{h \in C} \theta^{h}=0$.

Definition 3.6.2 restricts feasibility at date-event 0 to credible allocations. Only allocations that belong to the core of the ex-post economy are regarded as feasible.

Definition 3.6.3. Let some allocation and portfolio plan $(\bar{x}, \bar{\theta}) \in$ $X \times \Theta$ be given. A coalition $C \in \mathcal{C}$ can WSC-improve upon $(\bar{x}, \bar{\theta})$ at date-event $\bar{s} \in S^{\prime}$ if there exists a WSC-feasible $\left(x^{C}, \theta^{C}\right) \in X^{C} \times \Theta^{C}$ for $C$ at $\bar{s}=0$ or an ex post feasible $\left(x^{C}, \theta^{C}\right) \in X^{C} \times \Theta^{C}$ for $C$ at $\bar{s} \in S$ such that

$u^{h}\left(x^{h}\right)>u^{h}\left(\bar{x}^{h}\right), \quad h \in C$.

Definition 3.6.4. The Weak Sequential Core of the economy $\mathcal{E}$, denoted by $\operatorname{WSC}(\mathcal{E})$, is the collection of attainable allocations and portfolio plans $(\bar{x}, \bar{\theta}) \in X \times \Theta$ such that there is no date-event $\bar{S} \in S^{\prime}$ at which some coalition $C \in \mathcal{C}$ can WSC-improve upon $(\bar{x}, \bar{\theta})$.

There are a number of differences between our definition of WSC $(\&)$ and the definition of the Weak Sequential Core as given in Predtetchinski et al. (2006). In the latter paper the Weak Sequential Core is defined as an allocation $\bar{x}$ for which there is a portfolio plan that leads to a feasible intermediate allocation being such that, for every date-event $s \in S, \bar{x}_{s}$ belongs to the Classical Core of the corresponding ex-post economy. Moreover, there is no coalition $C$ that can block $\bar{x}$ by an allocation $x^{C}$ that is obtained in an analogous way. Our definition of $\operatorname{WSC}(\varepsilon)$ compares to the definition of the Weak Sequential Core as given in Predtetchinski et al. (2006) in the same way as our definition of $\operatorname{SSC}(\mathcal{E})$ compares to the definition of the Strong Sequential Core as given in Predtetchinski et al. (2002). The most substantial difference is that we do not require intermediate allocations to belong to $X$, for the same reasons as given before. Predtetchinski et al. (2006) only consider the case where the intermediate allocation does belong to $X$.

Example 3.3.4 (Continued). As before, it follows from Definition 3.1.2 that $\bar{x}_{s}^{h}=e_{s}^{h}$ for all $h \in H$ and $s \in S$ when $\bar{x} \in \operatorname{WSC}(\varepsilon)$. Since there are no assets, the Classical Core of all relevant ex-post economies is given by the no-trade allocation. Now it follows as before that

$\operatorname{WSC}(\varepsilon)=\{e\}$.

Our example illustrates that the Strong Sequential Core and the Classical Core have major flaws. The Strong Sequential Core is typically empty-valued, whereas the absence of asset markets does not matter in the Classical Core. The three other core concepts all correctly indicate that without commitment possibilities, no-trade is the only stable outcome.

\subsection{Projection}

Our example was characterized by the absence of assets. This feature facilitated the comparison of the various core concepts. To be able to compare the Classical Core to the other concepts in general, a projection function needs to be introduced, which projects an allocation and portfolio plan $(x, \theta) \in X \times \Theta$ to the allocation $x \in X$. The set of allocations that results after applying the projection function to a particular core concept is denoted by adding a star to the concept as a superscript; e.g. the set of allocations which belong to the Segregated Core is denoted by $\mathrm{SC}^{*}(\varepsilon)$. In the following we will compare how the various core concepts themselves, as well as their projections on allocations are related to one another.

\subsection{Basic core relationships}

In this subsection we present a number of relationships among the various core concepts that follow immediately from their definitions. The first two results are straightforward extensions of Observation 1 of Predtetchinski et al. (2006) to our set-up. Note that, compared to Observation 1 , we relax the assumptions on the utility functions, include the portfolio plan in the concepts, and we do not require the intermediate consumption bundle to lie in the consumption set.

Theorem 3.8.1. It holds that $\operatorname{SSC}(\varepsilon) \subset \operatorname{TSC}(\varepsilon)$.

Proof. Consider some $(\bar{x}, \bar{\theta}) \in \operatorname{SSC}(\varepsilon)$. If $\left(x^{C}, \theta^{C}\right) \in X^{C} \times \Theta^{C}$ is TSC-feasible at date-event 0 , then it is also SSC-feasible at dateevent 0 .

Theorem 3.8.2. It holds that $\operatorname{SSC}(\varepsilon) \subset \operatorname{WSC}(\varepsilon)$.

Proof. The proof follows immediately from the observation that WSC-feasibility for a coalition at date-event 0 implies SSCfeasibility for that coalition at date-event 0 . 
Finally, we had already argued that the Strong Sequential Core is a refinement of the Classical Core. This observation corresponds to Theorem 3 of Predtetchinski et al. (2002).

Theorem 3.8.3. It holds that $\operatorname{SSC}^{*}(\mathscr{E}) \subset \mathrm{CC}(\mathcal{E})$.

\section{Strongly complete markets}

In this section we analyze the various core concepts in a setting with strongly complete markets. Markets are strongly complete if for each commodity $l \in L$ and each date-event $s \in S$, there is a contract specifying the delivery of commodity $l$ contingent on the occurrence of date-event $s$; i.e. $\langle A\rangle=\mathbb{R}^{S L}$, where by $\langle A\rangle$ we denote the column space of the matrix $A$. Notice that the definition of strongly complete markets depends on the matrix $A$ only, and is independent of the price system.

This section is divided into two subsections; first we compare the various notions of the core under the assumptions made in Section 2. Surprisingly, the five notions of the core do not necessarily coincide, even when markets are strongly complete. Next we add some extra assumptions that make all concepts, except the Segregated Core, equivalent. Theorem 3 of Predtetchinski et al. (2002) provides conditions under which the Classical Core allocations coincide with the Strong Sequential Core allocations. Observation 2 of Predtetchinski et al. (2006) provides conditions under which their notions of the Two-stage Core, the Weak Sequential Core, and the Strong Sequential Core, i.e. notions where the intermediate consumption bundles are required to be feasible, lead to an equivalent set of allocations. The literature has not studied the relationships in combined allocation and portfolio space.

\subsection{General case}

In this subsection we first show that $\operatorname{SSC}(\mathscr{E})=\operatorname{TSC}(\mathscr{E}) \subset$ $\operatorname{WSC}(\varepsilon)$ and $\operatorname{CC}(\varepsilon) \supset \operatorname{SSC}^{*}(\mathscr{E})=\operatorname{TSC}^{*}(\mathscr{E}) \subset \operatorname{WSC}^{*}(\varepsilon)$. Next we argue by means of counterexamples that there are no further relationships. In particular this means that there are no general relationships between the Segregated Core and any of the other core concepts.

Theorem 4.1.1. When markets are strongly complete it holds that $\operatorname{SSC}(\varepsilon)=\operatorname{TSC}(\varepsilon) .^{3}$

Proof. The inclusion $\operatorname{SSC}(\mathscr{E}) \subset \mathrm{TSC}(\mathscr{E})$ follows immediately from Theorem 3.8.1.

Now we show that $\operatorname{TSC}(\varepsilon) \subset \operatorname{SSC}(\varepsilon)$. Consider some $(\bar{x}, \bar{\theta}) \in$ $\operatorname{TSC}(\varepsilon)$. Let $\left(x^{C}, \theta^{C}\right) \in X^{C} \times \Theta^{C}$ be SSC-feasible for coalition $C$ at date-event 0 . We construct $\tilde{\theta}^{C} \in \Theta^{C}$ such that $\left(x^{C}, \tilde{\theta}^{C}\right)$ is TSC-feasible for coalition $C$ at date-event 0 . Let $\hat{A}$ be a full-rank submatrix of $A$ and define, for $h \in C, \hat{\theta}^{h}=\hat{A}^{-1}\left(x_{-0}^{h}-e_{-0}^{h}\right)$. Notice that the existence of $\hat{A}$ follows from the fact that markets are strongly complete. It is immediate that $x_{s}^{h}=e_{s}^{h}+\hat{D}_{s} \hat{\theta}^{h}, s \in S$, where $\hat{D}_{s}$ is the submatrix of $D_{s}$ corresponding to $\hat{A}$, and $\sum_{h \in C} \hat{\theta}^{h}=$ 0 . We define $\tilde{\theta}^{C} \in \Theta^{C}$ as $\hat{\theta}^{C}$ extended by zeros in coordinates not corresponding to assets in $\hat{A}$. Then $\left(x^{C}, \tilde{\theta}^{C}\right)$ is TSC-feasible for coalition $C$ at date-event 0 . The proof is completed by realizing that if $C$ can SSC-improve at 0 using $(x, \theta)$, then $C$ can TSC-improve at 0 using $(x, \tilde{\theta})$.

The more difficult part of the proof of Theorem 4.1.1 is to show that $\operatorname{TSC}(\varepsilon) \subset \operatorname{SSC}(\varepsilon)$. Since both concepts coincide as far as blocking in period 1 is concerned, it only has to be shown that if $(x, \theta)$ is SSC-feasible for a coalition $C$ in period 0 , then there is a

\footnotetext{
3 This result extends Observation 2 in Predtetchinski et al. (2006).
}

portfolio plan $\tilde{\theta}$ such that $(x, \tilde{\theta})$ is TSC-feasible in period 0 . Even when markets are strongly complete, it is in general not the case that $(x, \theta)$ is TSC-feasible in period 0 itself. Indeed, there is no reason that $(x, \theta)$ satisfies Eq. (1) since the allocation $x$ might be quite different from the intermediate allocation induced by $\theta$. The portfolio plan $\tilde{\theta}$ therefore has to be chosen suitably.

We know from Theorem 3.8.2 that $\operatorname{SSC}(\mathscr{E}) \subset \mathrm{WSC}(\&)$. It is not necessarily the case though that $\operatorname{WSC}^{*}(\mathscr{E}) \subset \operatorname{SSC}^{*}(\mathscr{E})$, even when markets are strongly complete. The reason is that an allocation and portfolio plan that is SSC-feasible in period 0 may fail to induce allocations in the Classical Core of the resulting ex-post economies, and is therefore not WSC-feasible in period 0 . Indeed, there is nothing that precludes the Classical Core of a resulting ex-post economy to be empty. For the very same reason, it is not necessarily the case that $\mathrm{WSC}^{*}(\mathscr{E}) \subset \mathrm{CC}(\mathscr{E})$, even when markets are strongly complete.

Now we turn to the examination of the relation of the Classical Core to the other concepts. In the following theorems and examples we show that the Classical Core might not be restrictive enough, even in the case of strongly complete markets. We prove that there exist allocations in the Classical Core that do not belong to the Two-stage Core, to the Strong Sequential Core, or to the Weak Sequential Core. The Classical Core is basically a static concept, thus it does not take into account that certain allocations are unstable if further retrading is allowed for. We therefore argue that the Classical Core is not an appropriate concept in a dynamic setting even when markets are strongly complete.

Theorem 4.1.2. When markets are strongly complete it holds that $\operatorname{SSC}^{*}(\mathscr{E})=\operatorname{TSC}^{*}(\mathscr{E}) \subset \mathrm{CC}(\mathcal{E})$.

Proof. The equality is an immediate consequence of Theorem 4.1.1 and the inclusion follows from Theorem 3.8.3.

We show in the next example that the Two-stage Core, and so the Strong Sequential Core as well, can be a proper subset of the Classical Core. In the economy $\&$ of the example it holds that $\operatorname{SSC}^{*}(\varepsilon)=\operatorname{TSC}^{*}(\varepsilon) \subsetneq \mathrm{CC}(\varepsilon)$. The result is quite intuitive once one realizes that both in the Two-stage Core and in the Strong Sequential Core a coalition $C$ can redistribute the intermediate allocation $\left(e_{\bar{s}}^{h}+D_{\bar{s}} \bar{\theta}^{h}\right)_{h \in C}$ at date-event $\bar{s}$, while such is impossible in the case of the Classical Core. The construction used to demonstrate that a particular element of the Classical Core does not belong to the Two-stage Core also shows that it does neither belong to the Weak Sequential Core, nor the Segregated Core. In general it does not hold that $\mathrm{CC}(\mathcal{E}) \subset \operatorname{WSC}^{*}(\varepsilon)$ and it does not hold that $\mathrm{CC}(\varepsilon) \subset \mathrm{SC}^{*}(\varepsilon)$.

Example 4.1.3. Consider an economy $\&$ without uncertainty, three households, three commodities, and strongly complete markets, $S=\{1\}, H=\{1,2,3\}, L=\{1,2,3\}$, and $J=\{1,2,3\}$. The asset payoff matrix $A$ is given by

$A=\left(\begin{array}{lll}1 & 0 & 0 \\ 0 & 1 & 0 \\ 0 & 0 & 1\end{array}\right)$

The households' initial endowments are

$\left(e_{0}^{1}, e_{0}^{2}, e_{0}^{3}\right)=\left(\begin{array}{ccc}0 & 0 & 1 \\ 0 & 0 & 1 \\ \frac{1}{2} & \frac{1}{2} & 0\end{array}\right) \quad$ and $\quad\left(e_{1}^{1}, e_{1}^{2}, e_{1}^{3}\right)=\left(\begin{array}{lll}1 & 1 & 1 \\ 1 & 1 & 1 \\ 1 & 1 & 1\end{array}\right)$

We define the consumption sets as $X^{1}=X^{2}=X^{3}=\mathbb{R}_{+}^{3} \times\left(\mathbb{R}^{2} \times\right.$ $\left.\mathbb{R}_{+}\right)$. 


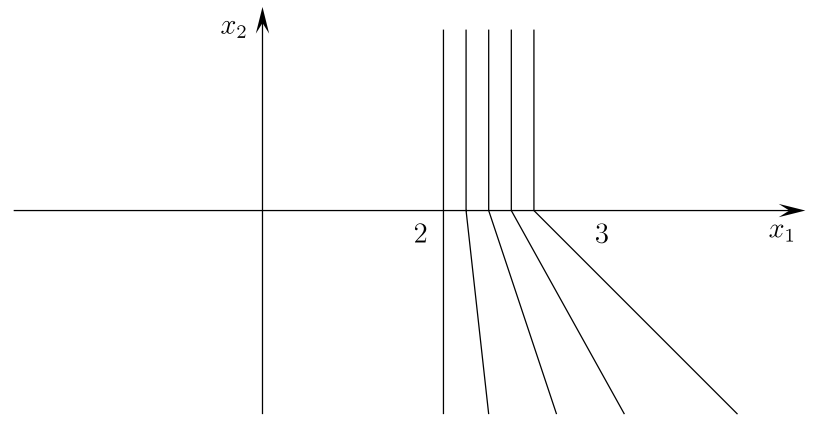

Fig. 1. Period 1 indifference curves for household 1.

The time-separable utility function $u^{1}$ satisfies $u^{1}\left(x^{1}\right)$

$$
= \begin{cases}x_{0,1}^{1}+x_{1,1}^{1} & \text { if } x_{1,1}^{1} \leq 2 \text { or }\left(x_{1,1}^{1}>2 \text { and } x_{1,2}^{1} \geq 0\right) \\ x_{0,1}^{1}+x_{1,1}^{1}+x_{1,2}^{1} & \text { if } x_{1,1}^{1}+x_{1,2}^{1} \geq 3 \text { and } x_{1,2}^{1} \leq 0 .\end{cases}
$$

For $x_{1,1}^{1}>2, x_{1,2}^{1}<0$, and $x_{1,1}^{1}+x_{1,2}^{1}<3, u^{1}$ is defined in such a way that it is continuous and strictly increasing in $x_{1,1}^{1}$ and $x_{1,2}^{1}$. Fig. 1 illustrates the indifference curves of household 1 in period 1 , given any amount of consumption in period 0 and any amount of consumption of commodity 3 in period 1 .

Similarly, $u^{2}$ satisfies

$$
\begin{aligned}
& u^{2}\left(x^{2}\right) \\
& \quad= \begin{cases}x_{0,2}^{2}+x_{1,2}^{2} & \text { if } x_{1,2}^{2} \leq 2 \text { or }\left(x_{1,2}^{2}>2 \text { and } x_{1,1}^{2} \geq 0\right) \\
x_{0,2}^{2}+x_{1,1}^{2}+x_{1,2}^{2} & \text { if } x_{1,1}^{2}+x_{1,2}^{2} \geq 3 \text { and } x_{1,1}^{2} \leq 0 .\end{cases}
\end{aligned}
$$

For $x_{1,2}^{2}>2, x_{1,1}^{2}<0$, and $x_{1,1}^{2}+x_{1,2}^{2}<3, u^{2}$ is defined in such a way that it is continuous and strictly increasing in $x_{1,1}^{2}$ and $x_{1,2}^{2}$.

Household 3 is only interested in commodity 3 and has utility function

$u^{3}\left(x^{3}\right)=x_{0,3}^{3}+x_{1,3}^{3}, \quad x^{3} \in X^{3}$.

We can easily compute that $u^{h}\left(e^{h}\right)=1$ for each household $h$.

Consider the following allocation,

$\left(\bar{x}_{0}^{1}, \bar{x}_{0}^{2}, \bar{x}_{0}^{3}\right)=\left(\begin{array}{lll}1 & 0 & 0 \\ 0 & 1 & 0 \\ 0 & 0 & 1\end{array}\right) \quad$ and $\quad\left(\bar{x}_{1}^{1}, \bar{x}_{1}^{2}, \bar{x}_{1}^{3}\right)=\left(\begin{array}{ccc}2 & 1 & 0 \\ 1 & 2 & 0 \\ 0 & 0 & 3\end{array}\right)$.

The resulting utilities are $u^{1}\left(\bar{x}^{1}\right)=u^{2}\left(\bar{x}^{2}\right)=3$, and $u^{3}\left(\bar{x}^{3}\right)=4$.

We claim that this allocation belongs to the Classical Core, but not to the Two-stage Core.

1. $\bar{x} \in \mathrm{CC}(\mathcal{E})$.

None of the singleton coalitions can block $\bar{x}$, since the utilities resulting from the initial endowments are strictly lower than $u^{h}\left(\bar{x}^{h}\right)$ for each household $h$. Also, no coalition involving household 3 can block the allocation, since household 3 cannot get utility higher than 4 .

Thus the only case to be checked is that of coalition $\{1,2\}$. Let $x^{\{1,2\}} \in X^{\{1,2\}}$ be CC-feasible for coalition $\{1,2\}$. We observe that $x_{0, l}^{h}=0, h=1,2, l=1,2$, and $\sum_{h \in\{1,2\}} x_{1, l}^{h}=2, l \in L$. For $x^{\{1,2\}}$ to block $\bar{x}$ it has to be the case that $u^{1}\left(x^{1}\right)>3$ and $u^{2}\left(x^{2}\right)>$ 3 , so $x_{1,1}^{1}>3$ and $x_{1,2}^{2}>3$, and consequently $x_{1,2}^{1}<-1$ and $x_{1,1}^{2}<-1$. Moreover, $u^{1}\left(x^{1}\right)>3$ and $u^{2}\left(x^{2}\right)>3$ now implies that $x_{1,1}^{1}+x_{1,2}^{1}>3$ and $x_{1,1}^{2}+x_{1,2}^{2}>3$. Since $u^{1}\left(x^{1}\right)=x_{1,1}^{1}+x_{1,2}^{1}$ and $u^{2}\left(x^{2}\right)=x_{1,1}^{2}+x_{1,2}^{2}, \sum_{h \in\{1,2\}} x_{1, l}^{h}=2, l \in L$, implies that the sum of the utilities of households 1 and 2 is therefore equal to 4 , leading to a contradiction.

Hence, the allocation $\bar{x}$ is an element of the Classical Core.
2. $\bar{x} \notin \operatorname{TSC}^{*}(\varepsilon) \cup \operatorname{WSC}^{*}(\varepsilon) \cup \operatorname{SC}^{*}(\varepsilon)$.

Suppose $\bar{\theta} \in \Theta$ is such that $(\bar{x}, \bar{\theta}) \in \operatorname{TSC}(\varepsilon) \cup \operatorname{WSC}(\varepsilon) \cup \operatorname{SC}(\varepsilon)$. Since coalition $\{3\}$ cannot block at date-event 1 , it holds that $e_{1,3}^{3}+\bar{\theta}_{3}^{3} \leq 3$, so $\bar{\theta}_{3}^{3} \leq 2$ and $\bar{\theta}_{3}^{1}+\bar{\theta}_{3}^{2} \geq-2$. The total resources for coalition $\{1,2\}$ at date-event 1 are

$\bar{y}=e_{1}^{1}+e_{1}^{2}+\bar{\theta}^{1}+\bar{\theta}^{2}$

Notice that $\bar{y}_{3} \geq 0$. It follows that $\left(x^{\{1,2\}}, \bar{\theta}^{\{1,2\}}\right) \in X^{\{1,2\}} \times \Theta^{\{1,2\}}$ given by $x_{0}^{h}=\overline{\bar{x}}_{0}^{h}, h=1,2$, and

$\left(x_{1}^{1}, x_{1}^{2}\right)=\left(\begin{array}{cc}2+\varepsilon & \bar{y}_{1}-2-\varepsilon \\ \bar{y}_{2}-2-\varepsilon & 2+\varepsilon \\ \bar{y}_{3} / 2 & \bar{y}_{3} / 2\end{array}\right)$

is ex post feasible for $\{1,2\}$ at date-event 1 . For $\varepsilon>0$ it holds that $x_{1, h}^{h}>2$. Since $x_{1, h}^{h}=2$ leads to a utility of 3 irrespective of the amounts consumed of the other date-event 1 commodities, and the utility function $u^{h}$ is strictly increasing in $x_{1, h}^{h}$, we have that $u^{h}\left(x^{h}\right)>3$ for both households, and so the allocation $\bar{x}$ can be blocked.

Our argument somewhat resembles the one of Roth and Postlewaite (1977), who pointed out that in a setting with indivisible commodities there are allocations in the Classical Core which are not part of the Classical Core when starting with that allocation as the initial endowment.

In the next example, we show that $\operatorname{SSC}^{*}(\varepsilon) \subset \mathrm{SC}^{*}(\mathcal{E})$ cannot hold in general. It follows that $\operatorname{TSC}^{*}(\varepsilon) \subset \mathrm{SC}^{*}(\mathscr{E})$ and $\mathrm{WSC}^{*}(\varepsilon) \subset$ $\mathrm{SC}^{*}(\&)$ cannot hold in general.

Example 4.1.4. Consider an economy $\&$ without uncertainty, three households, three commodities, and strongly complete markets, $S=\{1\}, H=\{1,2,3\}, L=\{1,2,3\}$, and $J=\{1,2,3\}$. The asset payoff matrix $A$ is given by

$A=\left(\begin{array}{lll}1 & 0 & 0 \\ 0 & 1 & 0 \\ 0 & 0 & 1\end{array}\right)$

The households' initial endowments are

$\left(e_{0}^{1}, e_{0}^{2}, e_{0}^{3}\right)=\left(\begin{array}{lll}1 & 0 & 0 \\ 0 & 1 & 0 \\ 0 & 0 & 1\end{array}\right) \quad$ and $\quad\left(e_{1}^{1}, e_{1}^{2}, e_{1}^{3}\right)=\left(\begin{array}{lll}1 & 1 & 1 \\ 1 & 1 & 1 \\ 1 & 1 & 1\end{array}\right)$.

We define the consumption sets as $X^{1}=X^{2}=\mathbb{R}_{+}^{3} \times\left(\mathbb{R}^{2} \times \mathbb{R}_{+}\right)$ and $X^{3}=\mathbb{R}_{+}^{3} \times \mathbb{R}^{3}$.

The utility functions are given by

$u^{1}\left(x^{1}\right)=x_{0,1}^{1}+x_{1,1}^{1}+\min \left\{0, x_{1,2}^{1}\right\}$,

$u^{2}\left(x^{2}\right)=x_{0,2}^{2}+x_{1,2}^{2}+\min \left\{0, x_{1,1}^{2}\right\}$,

$u^{3}\left(x^{3}\right)=x_{0,3}^{3}+x_{1,3}^{3}$.

We have that $u^{h}\left(e^{h}\right)=2$ for each household $h$.

Consider the following allocation,

$\left(\bar{x}_{0}^{1}, \bar{x}_{0}^{2}, \bar{x}_{0}^{3}\right)=\left(\begin{array}{lll}1 & 0 & 0 \\ 0 & 1 & 0 \\ 0 & 0 & 1\end{array}\right)$ and $\left(\bar{x}_{1}^{1}, \bar{x}_{1}^{2}, \bar{x}_{1}^{3}\right)=\left(\begin{array}{lll}2 & 1 & 0 \\ 1 & 2 & 0 \\ 0 & 0 & 3\end{array}\right)$.

The resulting utilities are $u^{1}\left(\bar{x}^{1}\right)=u^{2}\left(\bar{x}^{2}\right)=3$ and $u^{3}\left(\bar{x}^{3}\right)=4$.

We claim that this allocation belongs to the Classical Core, but not to the Segregated Core.

1. $\bar{x} \in \mathrm{CC}(\mathcal{E})$.

Clearly, none of the singleton coalitions can block the allocation $\bar{x}$, since $u^{h}\left(e^{h}\right)<u^{h}\left(\bar{x}^{h}\right)$ for each household $h$. Also, no coalition including household 3 can block the allocation, since there is no feasible allocation where household 3 gets utility exceeding 4 . We only have to verify that coalition $\{1,2\}$ cannot block $\bar{x}$. 
Suppose coalition $\{1,2\}$ blocks $\bar{x}$ by $x^{\{1,2\}} \in X^{\{1,2\}}$. It holds that $u^{1}\left(x^{1}\right)>3$ and $u^{2}\left(x^{2}\right)>3$, so $x_{0,1}^{1}+x_{1,1}^{1}+\min \left\{0, x_{1,2}^{1}\right\}>3$ and $x_{0,2}^{2}+x_{1,2}^{2}+\min \left\{0, x_{1,1}^{2}\right\}>3$. This leads to a contradiction since

$$
\begin{aligned}
& x_{0,1}^{1}+x_{1,1}^{1}+\min \left\{0, x_{1,2}^{1}\right\}+x_{0,2}^{2}+x_{1,2}^{2}+\min \left\{0, x_{1,1}^{2}\right\} \\
& \quad \leq x_{0,1}^{1}+x_{1,1}^{1}+x_{1,2}^{1}+x_{0,2}^{2}+x_{1,1}^{2}+x_{1,2}^{2} \\
& \quad \leq e_{0,1}^{1}+e_{1,1}^{1}+e_{1,2}^{1}+e_{0,2}^{2}+e_{1,1}^{2}+e_{1,2}^{2}=6 .
\end{aligned}
$$

Consequently, the allocation $\bar{x}$ is an element of the Classical Core.

2. $\bar{x} \in \operatorname{SSC}^{*}(\mathcal{E})$.

For $h \in H$, we define $\bar{\theta}^{h}=0$. We show that $(\bar{x}, \bar{\theta}) \in \operatorname{SSC}(\varepsilon)$. Since $\bar{x} \in C C(\mathscr{E})$, there is no coalition $C$ that can SSC-improve upon $\bar{x}$ at date-event 0 . It is straightforward to show that neither singleton coalitions, nor coalitions involving household 3 can SSC-improve upon $\bar{x}$ at date-event 1 .

It remains to be verified that coalition $\{1,2\}$ cannot SSCimprove upon $(\bar{x}, \bar{\theta})$ at date-event 1 . Suppose $\{1,2\}$ improves upon $(\bar{x}, \bar{\theta})$ at date-event 1 by $\left(x^{\{1,2\}}, \theta^{\{1,2\}}\right) \in X^{\{1,2\}} \times \Theta^{\{1,2\}}$. It should then be the case that $x_{1,1}^{1}+\min \left\{0, x_{1,2}^{1}\right\}>2$ and $x_{1,2}^{2}+$ $\min \left\{0, x_{1,1}^{2}\right\}>2$. It follows that $x_{1,1}^{1}>2$ and $x_{1,2}^{2}>2$, and by ex post feasibility that $x_{1,2}^{1}<0$ and $x_{1,1}^{2}<0$. The sum of period 1 utilities is therefore equal to $x_{1,1}^{1}+x_{1,2}^{1}+x_{1,1}^{2}+x_{1,2}^{2}>4$, whereas ex post feasibility at date-event 1 dictates this expression to be equal to 4 , a contradiction.

3. $\bar{x} \notin \mathrm{SC}^{*}(\varepsilon)$.

Suppose $\bar{\theta} \in \Theta$ is such that $(\bar{x}, \bar{\theta}) \in \operatorname{SC}(\mathcal{E})$.

(a) It holds that $\left(\left(\bar{x}_{0}^{3}, x_{1}^{3}\right), \bar{\theta}^{3}\right)$ is ex post feasible for coalition $\{3\}$ at date-event 1 if $x_{1}^{3}=e_{1}^{3}+\bar{\theta}^{3}$. To prevent coalition $\{3\}$ from blocking we need that

$\bar{\theta}_{3}^{3} \leq 2$.

(b) It holds that $\left(x^{\{1,2\}}, \theta^{\{1,2\}}\right) \in X^{\{1,2\}} \times \Theta^{\{1,2\}}$ is ex post feasible for coalition $\{1,2\}$ at date-event 1 if, for $h=1,2, x_{0}^{h}=$ $\bar{x}_{0}^{h}, \theta^{h}=\bar{\theta}^{h}$, and $\sum_{h \in\{1,2\}} x_{1}^{h}=e_{1}^{1}+e_{1}^{2}+\bar{\theta}^{1}+\bar{\theta}^{2}$. It follows that coalition $\{1,2\}$ can block at date-event 1 if

$\bar{\theta}_{1}^{1}+\bar{\theta}_{1}^{2}+\bar{\theta}_{2}^{1}+\bar{\theta}_{2}^{2}>0$ and $\bar{\theta}_{3}^{1}+\bar{\theta}_{3}^{2} \geq-2$.

To prevent coalition $\{1,2\}$ from blocking at date-event 1 we need

$\bar{\theta}_{1}^{1}+\bar{\theta}_{1}^{2}+\bar{\theta}_{2}^{1}+\bar{\theta}_{2}^{2} \leq 0$ or $\bar{\theta}_{3}^{1}+\bar{\theta}_{3}^{2}<-2$.

(c) It holds that $\left(x^{3}, \theta^{3}\right) \in X^{3} \times \Theta^{3}$ is SC-feasible for coalition $\{3\}$ at date-event 0 if $x_{0}^{3}=e_{0}^{3}, \theta^{3}=0$, and $x_{1}^{3}=\bar{x}_{1}^{3}-\bar{\theta}^{3}$. It follows that coalition $\{3\}$ can block at date-event 0 if $\bar{\theta}_{3}^{3}<0$. To prevent coalition $\{3\}$ from blocking at date-event 0 we need

$\bar{\theta}_{3}^{3} \geq 0$.

(d) It holds that $\left(x^{\{1,2\}}, \theta^{\{1,2\}}\right) \in X^{\{1,2\}} \times \Theta^{\{1,2\}}$ is SC-feasible for coalition $\{1,2\}$ at date-event 0 if, for $h=1,2, x_{0}^{h}=$ $\bar{x}_{0}^{h}, \theta^{1}+\theta^{2}=0, x_{1}^{1}=\bar{x}_{1}^{1}+\theta^{1}-\bar{\theta}^{1}$, and $x_{1}^{2}=\bar{x}_{1}^{2}+\theta^{2}-\bar{\theta}^{2}$. It follows that coalition $\{1,2\}$ can block at date-event 0 if $\bar{\theta}_{1}^{1}+\bar{\theta}_{1}^{2}+\bar{\theta}_{2}^{1}+\bar{\theta}_{2}^{2}<2$ and $\bar{\theta}_{3}^{1}+\bar{\theta}_{3}^{2} \leq 0$. Indeed, $\bar{\theta}_{3}^{1}+\bar{\theta}_{3}^{2} \leq 0$ means $\theta_{3}^{1}$ and $\theta_{3}^{2}$ can be chosen such that $x_{1,3}^{1} \geq 0$ and $x_{1,3}^{2} \geq 0$. Choose $\theta_{2}^{1}$ and $\theta_{1}^{2}$ such that $\theta_{2}^{1}<\bar{\theta}_{2}^{1}-1$ and $\theta_{1}^{2}<\bar{\theta}_{1}^{2}-1$, so $x_{1,2}^{1}<0$ and $x_{1,1}^{2}<0$. Moreover $\theta_{2}^{1}$ and $\theta_{1}^{2}$ can be chosen such that $\theta_{2}^{1}-\theta_{1}^{2}=\bar{\theta}_{1}^{1}+\bar{\theta}_{2}^{1}+\varepsilon-1$, where $\varepsilon$ is a given positive real number. We have that $u^{1}\left(x^{1}\right)=4-$ $\bar{\theta}_{1}^{1}-\bar{\theta}_{2}^{1}+\theta_{2}^{1}-\theta_{1}^{2}=3+\varepsilon$. Since $u^{2}\left(x^{2}\right)=4-\bar{\theta}_{1}^{2}-\bar{\theta}_{2}^{2}+\theta_{1}^{2}-\theta_{2}^{1}$, we find that $u^{1}\left(x^{1}\right)+u^{2}\left(x^{2}\right)>6$, so $u^{2}\left(x^{2}\right)>3$ when $\varepsilon$ is sufficiently small.

To prevent coalition $\{1,2\}$ from blocking at date-event 0 we therefore need

$\bar{\theta}_{1}^{1}+\bar{\theta}_{1}^{2}+\bar{\theta}_{2}^{1}+\bar{\theta}_{2}^{2} \geq 2$ or $\bar{\theta}_{3}^{1}+\bar{\theta}_{3}^{2}>0$.
From (2) it follows that the second part of the condition in (3) cannot hold, so we have $\bar{\theta}_{1}^{1}+\bar{\theta}_{1}^{2}+\bar{\theta}_{2}^{1}+\bar{\theta}_{2}^{2} \leq 0$. But this contradicts the first part of (5), thus $\bar{\theta}_{3}^{1}+\bar{\theta}_{3}^{2}>0$ follows. Then $\bar{\theta}_{3}^{3}=-\bar{\theta}_{3}^{1}-\bar{\theta}_{3}^{2}<0$, contradicting (4).

We show in the following example that the Segregated Core may contain allocations that are not individually rational.

Example 4.1.5. Consider an economy $\&$ without uncertainty, two households, two commodities, and strongly complete markets, $S=$ $\{1\}, H=\{1,2\}, L=\{1,2\}$, and $J=\{1,2\}$. The asset payoff matrix $A$ is given by

$A=\left(\begin{array}{ll}1 & 0 \\ 0 & 1\end{array}\right)$

The households' initial endowments are

$\left(e_{0}^{1}, e_{0}^{2}\right)=\left(\begin{array}{ll}0 & 1 \\ 1 & 0\end{array}\right)$ and $\left(e_{1}^{1}, e_{1}^{2}\right)=\left(\begin{array}{cc}0 & 0.9 \\ 1 & 0\end{array}\right)$.

We define the consumption sets as $X^{1}=X^{2}=\mathbb{R}_{+}^{2} \times \mathbb{R}_{+}^{2}$. The utility functions are given by

$u^{1}\left(x^{1}\right)=\sqrt{\left(x_{0,1}^{1}+1\right)\left(x_{1,1}^{1}+1\right)}+\sqrt{\left(x_{0,2}^{1}+1\right)\left(x_{1,2}^{1}+1\right)}$,

$u^{2}\left(x^{2}\right)=x_{0,2}^{2}+x_{1,2}^{2}$.

We have that $u^{1}\left(e^{1}\right)=3$ and $u^{2}\left(e^{2}\right)=0$.

Consider the following allocation,

$\left(\bar{x}_{0}^{1}, \bar{x}_{0}^{2}\right)=\left(\begin{array}{ll}1 & 0 \\ 0 & 1\end{array}\right)$ and $\left(\bar{x}_{1}^{1}, \bar{x}_{1}^{2}\right)=\left(\begin{array}{cc}0.9 & 0 \\ 0 & 1\end{array}\right)$

The resulting utilities are $u^{1}\left(\bar{x}^{1}\right)=\sqrt{2 \times 1.9}+1 \approx 2.9494$ and $u^{2}\left(\bar{x}^{2}\right)=2$.

We claim that this allocation belongs to the Segregated Core. For $h \in H$, we define $\bar{\theta}^{h}=0$. We show that $(\bar{x}, \bar{\theta}) \in \operatorname{SC}(\mathcal{E})$.

1. No SC-improvements at date-event 1.

According to Definition 3.1.2, the only ex post feasible consumption bundle for household 1 at date-event 1 is given by

$x_{0}^{1}=\left(\begin{array}{l}1 \\ 0\end{array}\right) \quad$ and $\quad x_{1}^{1}=\left(\begin{array}{l}0 \\ 1\end{array}\right)$,

which would result in a utility level of $2 \sqrt{2} \approx 2.8284<u^{1}\left(\bar{x}^{1}\right)$. Similarly, the only ex post feasible consumption bundle for household 2 at date-event 1 is given by

$x_{0}^{2}=\left(\begin{array}{l}0 \\ 1\end{array}\right)$ and $x_{1}^{2}=\left(\begin{array}{c}0.9 \\ 0\end{array}\right)$,

which would result in a utility level of $1<u^{2}\left(\bar{x}^{2}\right)$. Ex post feasibility for coalition $\{1,2\}$ at date-event 1 leads to allocations with $x_{1,2}^{2} \leq 1$, so it is impossible to SC-improve upon the utility of household 2 at date-event 1 .

2. No SC-improvements at date-event 0 .

According to Definition 3.3.1, the only SC-feasible consumption bundle for household 1 at date-event 0 is given by

$x_{0}^{1}=\left(\begin{array}{l}0 \\ 1\end{array}\right)$ and $x_{1}^{1}=\left(\begin{array}{c}0.9 \\ 0\end{array}\right)$,

which results in a utility level of $\sqrt{1.9}+\sqrt{2} \approx 2.7926<u^{1}\left(\bar{x}^{1}\right)$. Similarly, the only SC-feasible consumption bundle for household 2 at date-event 0 is given by

$x_{0}^{2}=\left(\begin{array}{l}1 \\ 0\end{array}\right)$ and $x_{1}^{2}=\left(\begin{array}{l}0 \\ 1\end{array}\right)$,

which results in a utility level of $1<u^{2}\left(\bar{x}^{2}\right)$. 
SC-feasibility for coalition $\{1,2\}$ at date-event 0 implies $x_{0,2}^{2} \leq$ 1 and $x_{1,2}^{2} \leq 1$, so it is impossible to SC-improve upon the utility of household 2 at date-event 0 .

The allocation $\bar{x}$ in this example cannot belong to any of the other cores. Indeed, consider any $\bar{\theta} \in \Theta$ such that $\sum_{h \in H} \bar{\theta}^{h}=$ 0 , so $(\bar{x}, \bar{\theta})$ is attainable. Since $u^{1}\left(\bar{x}^{1}\right)<u^{1}\left(e^{1}\right)$ and $\left(e^{1}, 0\right)$ is SSC-feasible, WSC-feasible, and TSC-feasible for household 1 at date-event 0 , household 1 can block $(\bar{x}, \bar{\theta})$ at date-event 0 . It is also obvious for the same reason that $\bar{x}$ does not belong to the Classical Core. The example also shows that the Segregated Core is problematic, as individual rationality is a property that should be satisfied by a reasonable core concept.

Fig. 2 summarizes the relationships that we have found in this section.

\subsection{Some extra assumptions}

In this subsection we introduce two extra assumptions that guarantee all core concepts to coincide when markets are strongly complete, with the exception of the Segregated Core that contains all the other ones.

Assumption 4.2.1. For all $h \in H$, it holds that $e^{h} \in X^{h}$.

Assumption 4.2.2. Let $\varepsilon_{s, X^{c}, \theta^{c}}=\left(X_{s}^{h}, \bar{e}_{s}^{h}, u_{\mid X_{s}^{h}}^{h}\right)_{h \in C}$ be an ex-post economy with, for $h \in C, \bar{e}_{s}^{h} \in X^{h}$ and $u^{h}\left(\bar{e}_{s}^{h}\right) \geq u^{h}\left(e^{h}\right)$. Then $\mathrm{CC}\left(\varepsilon_{s, x^{C}, \theta^{C}}\right) \neq \emptyset$.

This assumption would for instance be satisfied if consumption sets are bounded from below and utility functions are quasiconcave.

Assumption 4.2.3. The set of Strongly Pareto Optimal allocations of the economy $\&$ coincides with the set of Weakly Pareto Optimal allocations of $\mathcal{E}$.

It is also not difficult to make assumptions on the primitives such that this assumption is satisfied, for instance the assumption that the utility function is strictly monotonic.

Under these extra assumptions it can be shown that the set of allocations which belong to the Strong Sequential Core and to the Weak Sequential Core coincide with one another.

Theorem 4.2.4. When markets are strongly complete it holds under Assumption 4.2.2 that $\operatorname{SSC}(\varepsilon)=\operatorname{WSC}(\varepsilon)$.

Proof. By Theorem 3.8.2 it holds that $\operatorname{SSC}(\mathscr{E}) \subset \mathrm{WSC}(\mathscr{E})$.

We show next that $\operatorname{WSC}(\mathscr{E}) \subset \operatorname{SSC}(\mathscr{E})$. Consider $(\bar{x}, \bar{\theta}) \in$ $\operatorname{WSC}(\mathscr{E})$. Let $C \in \mathcal{C}$ be a coalition that SSC-improves upon $(\bar{x}, \bar{\theta})$ by $\left(x^{C}, \theta^{C}\right) \in X^{C} \times \Theta^{C}$ at date-event 0 . We show that coalition $C$ can WSC-improve upon $(\bar{x}, \bar{\theta})$ at date-event 0 by some $\left(\hat{x}^{C}, \hat{\theta}^{C}\right)$, which leads to a contradiction since $(\bar{x}, \bar{\theta}) \in \operatorname{WSC}(\mathcal{E})$. According to Definition 3.5.1,

$$
\begin{aligned}
& \sum_{h \in C} x^{h}=\sum_{h \in C} e^{h}, \\
& \sum_{h \in C} \theta^{h}=0 .
\end{aligned}
$$

Since markets are strongly complete we can choose $\hat{\theta}^{C} \in \Theta^{C}$ such that

$x_{-0}^{h}=e_{-0}^{h}+A \hat{\theta}^{h}, \quad h \in C$,

$\sum_{h \in C} \hat{\theta}^{h}=0$.

We claim that $\operatorname{CC}\left(\varepsilon_{s, x^{C}, \hat{\theta}^{C}}\right)$ is non-empty for every $s \in S$. Notice that $\bar{e}_{s, s}^{h}=e_{s}^{h}+D_{s} \hat{\theta}^{h}=x_{s}^{h}$ and $\bar{e}_{s,-s}^{h}=x_{-s}^{h}$, so $\bar{e}_{s}^{h}=x^{h} \in X^{h}$. Since $(\bar{x}, \bar{\theta}) \in$ WSC $(\mathcal{E})$, it cannot be WSC-improved upon at date-event 0 by any coalition $\{h\}$, so $u^{h}\left(\bar{x}^{h}\right) \geq u^{h}\left(e^{h}\right), h \in H$. Since coalition $C$ SSC-improves upon $(\bar{x}, \bar{\theta})$ at date-event 0 by $\left(x^{C}, \theta^{C}\right)$, we have $u^{h}\left(\bar{e}_{s}^{h}\right)=u^{h}\left(x^{h}\right)>u^{h}\left(\bar{x}^{h}\right) \geq u^{h}\left(e^{h}\right), h \in C$. By Assumption 4.2.2, $\operatorname{CC}\left(\varepsilon_{s, x^{C}, \hat{\theta}^{c}}\right) \neq \emptyset$. For $s \in S, h \in C$, we choose $\hat{x}_{s}^{h}$ corresponding to

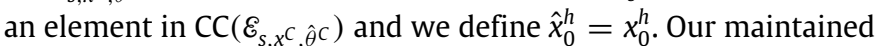
assumption that utility functions are state-separable implies that $\hat{x}^{C} \in \mathrm{CC}\left(\mathcal{E}_{s, \hat{x}^{C}, \hat{\theta}^{C}}\right)$. It follows that $(\hat{x}, \hat{\theta})$ is WSC-feasible for $C$ at dateevent 0 . Since

$$
\begin{aligned}
u^{h}\left(\bar{x}^{h}\right)<u^{h}\left(x^{h}\right) & =\sum_{s \in S} u_{s}^{h}\left(x_{0}^{h}, x_{s}^{h}\right) \leq \sum_{s \in S} u_{s}^{h}\left(\hat{x}_{0}^{h}, \hat{x}_{s}^{h}\right) \\
& =u^{h}\left(\hat{x}^{h}\right), \quad h \in C,
\end{aligned}
$$

it is also a WSC-improvement.

By Theorem 3.8.2 it holds that $\operatorname{SSC}(\varepsilon) \subset \operatorname{WSC}(\varepsilon)$. The other direction, $\operatorname{WSC}(\varepsilon) \subset \operatorname{SSC}(\varepsilon)$ is more difficult to obtain, since a particular SSC-improvement need not be a WSC-improvement. Nevertheless, in case there is an SSC-improvement, it is always possible to find some WSC-improvement.

The following theorem shows that under Assumption 4.2.3 the Classical Core coincides with the Strong Sequential Core. Note that this result was stated in Theorem 3 of Predtetchinski et al. (2002) under somewhat stronger assumptions.

Theorem 4.2.5. When markets are strongly complete it holds under Assumption 4.2.3 that $\operatorname{SSC}^{*}(\varepsilon)=\mathrm{CC}(\mathcal{E})$.

Proof. By Theorem 4.1.2 it holds that $\operatorname{SSC}^{*}(\&) \subset \mathrm{CC}(\&)$.

We show next that $\mathrm{CC}(\mathcal{E}) \subset \operatorname{SSC}^{*}(\varepsilon)$. Let $\bar{x}$ belong to $\mathrm{CC}(\varepsilon)$. Since markets are strongly complete, there is $\bar{\theta} \in \Theta$ such that $\bar{x}_{-0}^{h}=e_{-0}^{h}+A \bar{\theta}^{h}$ and $\sum_{h \in H} \bar{\theta}^{h}=0$.

We show that $(\bar{x}, \bar{\theta}) \in \operatorname{SSC}(\mathcal{E})$. Suppose that there is a dateevent $s \in S$ at which a coalition $C \in \mathcal{C}$ can SSC-improve upon $(\bar{x}, \bar{\theta})$ by $\left(x^{C}, \theta^{C}\right) \in X^{C} \times \Theta^{C}$. For $h \notin C$ we define

$x^{h}=\bar{x}^{h}$.

It follows from the fact that $C$ SSC-improves upon $(\bar{x}, \bar{\theta})$ by $\left(x^{C}, \theta^{C}\right)$ that

$u^{h}\left(x^{h}\right)>u^{h}\left(\bar{x}^{h}\right), \quad h \in C$,

$u^{h}\left(x^{h}\right)=u^{h}\left(\bar{x}^{h}\right), \quad h \notin C$.

Moreover,

$\sum_{h \in H} x_{-s}^{h}=\sum_{h \in H} \bar{x}_{-s}^{h}=\sum_{h \in H} e_{-s}^{h}$,

$\sum_{h \in H} x_{s}^{h}=\sum_{h \in C}\left(e_{s}^{h}+D_{s} \bar{\theta}^{h}\right)+\sum_{h \in H \backslash C}\left(e_{s}^{h}+D_{s} \bar{\theta}^{h}\right)=\sum_{h \in H} e_{s}^{h}$,

so $x$ is an attainable allocation. Hence, $\bar{x}$ is not strongly Pareto optimal, therefore by Assumption 4.2.3 not weakly Pareto optimal, so does not belong to $\mathrm{CC}(\mathcal{E})$, a contradiction. Consequently, there is no coalition $C \in \mathcal{C}$ that can SSC-improve upon $(\bar{x}, \bar{\theta})$ at a dateevent $s \in S$.

Since SSC-feasibility at date-event 0 is equivalent to CCfeasibility, there is no coalition $C \in \mathcal{C}$ that can SSC-improve upon $(\bar{x}, \bar{\theta})$ at date-event 0 .

It follows that $(\bar{x}, \bar{\theta}) \in \operatorname{SSC}(\varepsilon)$.

When we employ Assumption 4.2.3 we can also obtain a definite relationship between the Classical Core and the Segregated Core, and therefore between all the other core concepts and the Segregated Core. The Segregated Core unequivocally contains the other concepts.

Theorem 4.2.6. When markets are strongly complete it holds under Assumption 4.2.3 that $\mathrm{CC}(\mathcal{E}) \subset \mathrm{SC}^{*}(\mathcal{E})$. 

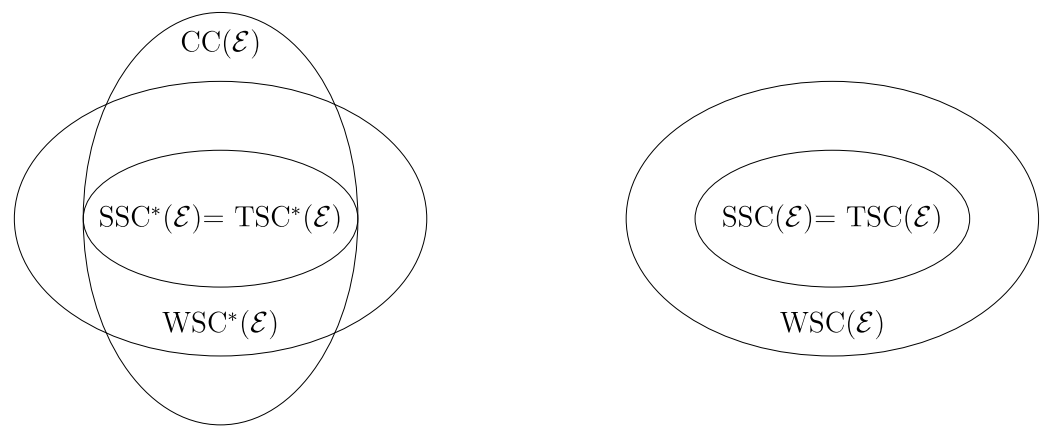

Fig. 2. Relationship of the core concepts when markets are strongly complete.

Proof. Let $\bar{x}$ belong to $\mathrm{CC}(\mathcal{E})$. Since markets are strongly complete we can choose $\bar{\theta}$ such that $\bar{x}_{-0}^{h}=e_{-0}^{h}+A \bar{\theta}^{h}$ and $\sum_{h \in H} \bar{\theta}^{h}=0$. We show that $(\bar{x}, \bar{\theta}) \in \mathrm{SC}(\mathcal{E})$.

Suppose that there is a date-event $s \in S$ at which a coalition $C \in \mathcal{C}$ can SC-improve upon $(\bar{x}, \bar{\theta})$ by $\left(x^{C}, \theta^{C}\right) \in X^{C} \times \Theta^{C}$.

We define

$x^{h}=\bar{x}^{h}, \quad h \notin C$.

It follows from the fact that C SC-improves upon $(\bar{x}, \bar{\theta})$ by $\left(x^{C}, \theta^{C}\right)$ at date-event $s$ that

$u^{h}\left(x^{h}\right)>u^{h}\left(\bar{x}^{h}\right), \quad h \in C$,

$u^{h}\left(x^{h}\right)=u^{h}\left(\bar{x}^{h}\right), \quad h \notin C$.

Moreover,

$\sum_{h \in H} x_{s}^{h}=\sum_{h \in C}\left(e_{s}^{h}+D_{s} \bar{\theta}^{h}\right)+\sum_{h \in H \backslash C}\left(e_{s}^{h}+D_{s} \bar{\theta}^{h}\right)=\sum_{h \in H} e_{s}^{h}$,

so $x$ is an attainable allocation.

Hence, $\bar{x}$ is not strongly Pareto optimal, therefore not weakly Pareto optimal by Assumption 4.2.3, so does not belong to $\mathrm{CC}(\mathcal{E})$, a contradiction. Consequently, there is no coalition $C \in \mathcal{C}$ that can SC-improve upon $(\bar{x}, \bar{\theta})$ at a date-event $s \in S$.

Suppose there is a coalition $C$ that can SC-improve upon $(\bar{x}, \bar{\theta})$ by $\left(x^{C}, \theta^{C}\right) \in X^{C} \times \Theta^{C}$ at date-event 0 . For every date-event $s \in S$,

$$
\begin{aligned}
\sum_{h \in C} x_{s}^{h} & =\sum_{h \in C}\left(\bar{x}_{s}^{h}+D_{s}\left(\theta^{h}-\bar{\theta}^{h}\right)\right) \\
& =\sum_{h \in C}\left(e_{s}^{h}+D_{s} \theta^{h}\right) \\
& =\sum_{h \in C} e_{s}^{h},
\end{aligned}
$$

whereas $\sum_{h \in C} x_{0}^{h}=\sum_{h \in C} e_{0}^{h}$. It follows that $x^{C}$ is CC-feasible for coalition $C$, so coalition $C$ can CC-improve upon $\bar{x}$ by $x^{C}$, a contradiction to $\bar{x} \in \mathrm{CC}(\mathcal{E})$.

It follows that $(\bar{x}, \bar{\theta}) \in \mathrm{SC}(\varepsilon)$.

One may wonder about the reverse relationship, i.e. is it possible to show that under Assumptions 4.2.2 and 4.2.3 the Segregated Core coincides with the Strong Sequential Core? Notice that Example 4.1.5 demonstrates that the Segregated Core may contain allocations that are not individually rational. Example 4.1.5 satisfies Assumption 4.2.2, but not Assumption 4.2.3. However, it can easily be modified to satisfy the latter assumption as well. Indeed, when for $\varepsilon>0$ sufficiently small we define

$u^{2}\left(x^{2}\right)=\varepsilon\left(x_{0,1}^{2}+x_{1,1}^{2}\right)+x_{0,2}^{2}+x_{1,2}^{2}$,

then Assumption 4.2.3 is satisfied. Now it can be verified that the not individually rational allocation $\bar{x}$ still belongs to $\mathrm{SC}^{*}(\mathcal{E})$. Clearly, such an allocation cannot belong to any of the other cores.

Another issue is whether the result can be extended to the statement $\operatorname{SSC}(\&) \subset \operatorname{SC}(\&)$. It is not hard to construct examples satisfying Assumptions 4.2.2 and 4.2.3 such that $\operatorname{SSC}(\mathcal{E}) \backslash \mathrm{SC}(\mathscr{E}) \neq$ $\emptyset$. The reason is that $\left(x^{C}, \theta^{C}\right) \in X^{C} \times \Theta^{C}$ may be SC-feasible for coalition $C \in \mathcal{C}$ at date-event 0 , but not SSC-feasible for that coalition at that date-event. Indeed, when defining SC-feasibility at date-event 0 , coalition $C$ expects net trades at date-events in $S$ not to be affected. The sum of these net trades over the coalition members is not equal to zero in general, so the coalition members do in general not expect that the sum of their consumption bundles in period 1 is equal to $\sum_{h \in C} e_{-0}^{h}$, unlike the case of SSC-feasibility. When markets are strongly complete it is true that $\operatorname{SC}^{*}(\mathscr{E})$ contains $\operatorname{SSC}^{*}(\varepsilon)$, but the way these allocations are supported, i.e. the choice of $\bar{\theta}$ may well be different.

We summarize the results in this subsection in Fig. 3.

\section{Finance economies and incomplete markets}

In this section we generalize the strongly complete market structure to an arbitrary market structure for the case of finance economies, i.e. there is one commodity per date-event.

For some results in this section, we will make use of the following minor additional assumption.

Assumption 5.1. For $h \in H, u^{h}$ is increasing.

Since by Assumption 2.1 the utility function is non-satiated in every date-event and since we are considering economies with one commodity per date-event in this section, Assumption 5.1 is only made to rule out the case where utility functions are decreasing.

The Classical Core of an ex-post finance economy is nonempty if the initial endowments belong to the consumption set. In particular, it follows that Assumptions 5.1 and 4.2 .1 imply Assumption 4.2.2. Under this assumption WSC-blocking becomes easier and we can show that the Weak Sequential Core is a subset of the Two-stage Core. Since in finance economies there are no gains from trade in ex-post economies, we can even show that the two concepts coincide.

Theorem 5.2. When $\varepsilon$ is a finance economy it holds under Assumptions 5.1 and 4.2.1 that $\operatorname{WSC}(\mathcal{E})=\operatorname{TSC}(\mathcal{E})$.

Proof. The inclusion $\operatorname{WSC}(\varepsilon) \subset \operatorname{TSC}(\mathscr{E})$ will follow from Theorem 7.1, where we treat the case with multiple commodities per date-event.

Consider $(\bar{x}, \bar{\theta}) \in \operatorname{TSC}(\mathcal{E})$. Let $\left(x^{C}, \theta^{C}\right)$ be WSC-feasible for coalition $C \in \mathcal{C}$ at date-event 0 , so

$x^{C} \in \mathrm{CC}\left(\varepsilon_{s, x^{C}, \theta^{C}}\right), \quad s \in S$.

We argue that, for $h \in C$,

$x_{s}^{h}=e_{s}^{h}+D_{s} \theta^{h}, \quad s \in S$.

Suppose that there is $h^{\prime} \in C$ and $s \in S$ such that

$x_{s}^{h^{\prime}} \neq e_{s}^{h^{\prime}}+D_{s} \theta^{h^{\prime}}$. 

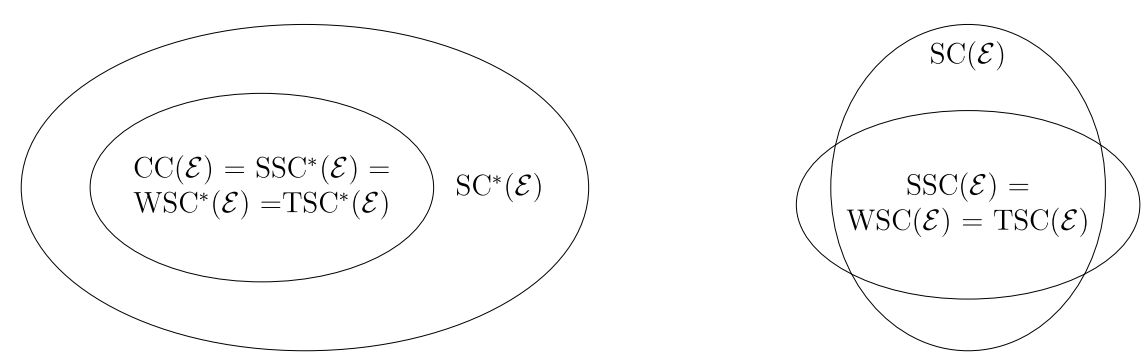

Fig. 3. Relationship of the core concepts when markets are strongly complete-with extra assumptions.

Since $\sum_{h \in C} x^{h}=\sum_{h \in C} e^{h}, h^{\prime}$ can be chosen to satisfy

$x_{s}^{h^{\prime}}<e_{s}^{h^{\prime}}+D_{s} \theta^{h^{\prime}}$.

Obviously, this contradicts $x^{C} \in \mathrm{CC}\left(\varepsilon_{s, x^{C}, \theta^{C}}\right)$ as local non-satiation at date-event $s$ of the increasing function $u^{h^{\prime}}$ implies that coalition $\left\{h^{\prime}\right\}$ would block. Consequently, we have shown that (6) holds. It follows that $\left(x^{C}, \theta^{C}\right)$ is TSC-feasible for coalition $C$ at date-event 0 .

Observation 3 of Predtetchinski et al. (2006) provides slightly stronger conditions for finance economies under which the set of Two-stage Core allocations coincides with the set of Weak Sequential Core allocations when the intermediate consumption bundles are required to be feasible.

The next result establishes the equivalence of the Two-stage Core and the Segregated Core, and, in the light of Theorem 5.2 the equivalence of the Weak Sequential Core and the Segregated Core.

Theorem 5.3. When $\varepsilon$ is a finance economy it holds under 4.2 .1 and Assumption 5.1 that $\operatorname{TSC}(\mathscr{E})=\operatorname{SC}(\varepsilon)$.

Proof. Let $(\bar{x}, \bar{\theta})$ be an element of $\operatorname{TSC}(\mathcal{E})$. We argue that, for $h \in$ $H, \bar{x}_{-0}^{h}=e_{-0}^{h}+A \bar{\theta}^{h}$. Suppose that there is $h \in H$ and $s \in S$ such that

$\bar{\chi}_{s}^{h} \neq e_{s}^{h}+D_{s} \bar{\theta}^{h}$.

Attainability of $(\bar{x}, \bar{\theta})$ implies that $h$ can be chosen to satisfy

$\bar{x}_{s}^{h}<e_{s}^{h}+D_{s} \bar{\theta}^{h}$.

Now $\left(x^{h}, \bar{\theta}^{h}\right) \in X^{h} \times \Theta^{h}$ defined by $x_{s}^{h}=e_{s}^{h}+D_{s} \bar{\theta}^{h}$ and $x_{-s}^{h}=\bar{x}_{-s}^{h}$ is ex post feasible for $\{h\}$ at date-event $s$ and satisfies $u^{h}\left(x^{h}\right)>u^{h}\left(\bar{x}^{h}\right)$ by local non-satiation at date-event $s$ of the increasing function $u^{h}$, which contradicts that $(\bar{x}, \bar{\theta})$ belongs to $\operatorname{TSC}(\mathcal{E})$. Consequently, for every $h \in H$ and $s \in S$ it holds that

$\bar{x}_{s}^{h}=e_{s}^{h}+D_{s} \bar{\theta}^{h}$.

Let $\left(x^{C}, \theta^{C}\right)$ be SC-feasible for coalition $C \in \mathcal{C}$ at date-event 0 . For $h \in C, s \in S$, it follows that $x_{s}^{h}-\bar{x}_{s}^{h}=D_{s}\left(\theta^{h}-\bar{\theta}^{h}\right)$, so

$$
\begin{aligned}
x_{s}^{h} & =\bar{x}_{s}^{h}+D_{s}\left(\theta^{h}-\bar{\theta}^{h}\right) \\
& =e_{s}^{h}+D_{s} \theta^{h},
\end{aligned}
$$

where the last equality follows from (7). It is now immediate that $\left(x^{C}, \theta^{C}\right)$ is TSC-feasible for coalition $C$ at date-event 0 . It follows that $(\bar{x}, \bar{\theta}) \in \operatorname{SC}(\varepsilon)$.

Let $(\bar{x}, \bar{\theta})$ be an element of $\mathrm{SC}(\mathcal{E})$. It follows by exactly the same argument as in the first part of the proof that, for $h \in H, \bar{x}_{-0}^{h}=$ $e_{-0}^{h}+A \bar{\theta}^{h}$. Let $\left(x^{C}, \theta^{C}\right)$ be TSC-feasible for coalition $C \in \mathcal{C}$ at dateevent 0 . For $h \in C, s \in S$, it follows that $x_{s}^{h}=e_{s}^{h}+D_{s} \theta^{h}$, so

$$
\begin{aligned}
x_{s}^{h}-\bar{x}_{s}^{h} & =e_{s}^{h}+D_{s} \theta^{h}-e_{s}^{h}-D_{s} \bar{\theta}^{h} \\
& =D_{s}\left(\theta^{h}-\bar{\theta}^{h}\right) .
\end{aligned}
$$

It is now immediate that $\left(x^{C}, \theta^{C}\right)$ is SC-feasible for coalition $C$ at date-event 0 . It follows that $(\bar{x}, \bar{\theta}) \in \operatorname{TSC}(\mathscr{E})$.
Even in a finance economy, when markets are not strongly complete, the Two-stage Core and the Strong Sequential Core do not coincide anymore. Theorem 3.8.1 demonstrates that the inclusion $\operatorname{SSC}(\mathscr{E}) \subset \mathrm{TSC}(\mathscr{E})$ still holds. The example of Section 3 shows that in a finance economy without asset markets the Strong Sequential Core is typically empty, whereas the Segregated Core is equal to the initial endowments.

Without the assumption of strongly complete markets, the Classical Core is unrelated to the Weak Sequential Core, the Twostage Core, and the Segregated Core. In the example used in Section 3 of a finance economy without asset markets, the latter three core concepts coincide with the initial endowments. Only in the extreme case where the initial endowments are Pareto efficient, the Classical Core will be equal to the initial endowments. It is immediate that the Strong Sequential Core is a subset of the Classical Core, as it also follows from Theorem 3.8.3.

Fig. 4 summarizes the results for the two-period finance economies and a general market structure.

\section{Competitive equilibrium and the core}

Before studying the relationship of the various core concepts for the general case - multiple commodities and an arbitrary market structure - we first address the question whether the competitive equilibrium belongs to a particular notion of the core.

Definition 6.1. A competitive equilibrium for an economy $\mathcal{E}$ is an element $\left(x^{*}, \theta^{*}, p^{*}, q^{*}\right) \in X \times \Theta \times \mathbb{R}^{S^{\prime} L} \times \mathbb{R}^{J}$ that satisfies the following conditions:

1. For $h \in H$,

$$
\begin{aligned}
& \left(x^{* h}, \theta^{* h}\right) \in \arg \max _{\left(x^{h}, \theta^{h}\right) \in X^{h} \times \Theta^{h}} u^{h}\left(x^{h}\right) \\
& \text { s.t. } p_{0}^{*} x_{0}^{h}+q^{*} \theta^{h}=p_{0}^{*} e_{0}^{h}, \\
& p_{s}^{*} x_{s}^{h}=p_{s}^{*}\left(e_{s}^{h}+D_{s} \theta^{h}\right), \quad s \in S,
\end{aligned}
$$

2.

$$
\sum_{h \in H} x^{* h}=\sum_{h \in H} e^{h},
$$

3.

$$
\sum_{h \in H} \theta^{* h}=0
$$

Since we have assumed local non-satiation at every date-event, we can state all budget relations with equality as far as equilibrium is concerned.

By $p_{-0} \square x_{-0}^{h}$ we denote the vector $\left(p_{s} x_{s}^{h}\right)_{s \in S} \in \mathbb{R}^{S}$ and by $p_{-0} \square A$ we denote the $(S \times J)$-matrix whose $j$-th column is $p_{-0} \square A^{j}$. We define the budget set of household $h$ as

$$
\begin{aligned}
B^{h}(p, q)= & \left\{\left(x^{h}, \theta^{h}\right) \in X^{h} \times \Theta^{h} \mid p_{0} x_{0}^{h}+q \theta^{h} \leq p_{0} e_{0}^{h}\right. \\
& \text { and } \left.p_{-0} \square x_{-0}^{h} \leq p_{-0} \square\left(e_{-0}^{h}+A \theta^{h}\right)\right\} .
\end{aligned}
$$



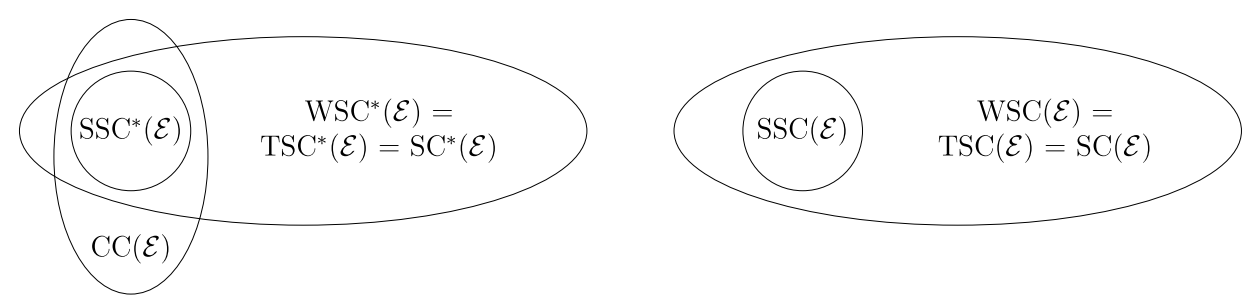

Fig. 4. Relationship of the core concepts in finance economies.

Markets are complete at prices $p$ if $\left\langle p_{-0} \square A\right\rangle=\mathbb{R}^{S}$ and are said to be incomplete otherwise. Note that when $\left\langle p_{-0}^{*} \square A\right\rangle$ has full dimension, then Definition 6.1 reduces to the definition of an Arrow-Debreu equilibrium.

The following result, equilibrium asset prices are compatible with a strictly positive state price vector, is well-known. The proof, though standard, is provided since our assumptions on the primitives are weaker than what is usually found in the literature.

Theorem 6.2. Let $\left(x^{*}, \theta^{*}, p^{*}, q^{*}\right)$ be a competitive equilibrium. Then there exists a strictly positive state price vector $\pi \in \mathbb{R}_{++}^{S}$ such that

$q^{*}=\pi\left(p_{-0}^{*} \square A\right)$.

Proof. We first recall Stiemke's Lemma: Let $p_{-0} \in \mathbb{R}^{S L}$ and $q \in$ $\mathbb{R}^{J}$ be given. There does not exist a portfolio $\theta^{h} \in \mathbb{R}^{J}$ such that $\left(p_{-0} \square A\right) \theta^{h} \geq 0$ and $q \theta^{h} \leq 0$ with at least one strict inequality if and only if there exists a strictly positive state price vector $\pi \in$ $\mathbb{R}_{++}^{S}$ such that $q=\pi\left(p_{-0} \square A\right)$.

All that remains to be shown is that at equilibrium $\left(x^{*}, \theta^{*}, p^{*}, q^{*}\right)$ there does not exist a portfolio $\theta^{h} \in \mathbb{R}^{J}$ such that $\left(p_{-0}^{*} \square A\right) \theta^{h} \geq 0$ and $q^{*} \theta^{h} \leq 0$ with at least one strict inequality. Suppose that such a portfolio exists and let date-event $s \in S^{\prime}$ carry a strict inequality. Since the utility function of a household is locally nonsatiated at date-event $s$, the equilibrium choice of the household is not maximizing utility, a contradiction.

Now we show that the competitive equilibrium belongs to the Segregated Core, thereby reproducing the result of Bester (1984) in our set-up.

Theorem 6.3. Let $\left(x^{*}, \theta^{*}, p^{*}, q^{*}\right)$ be a competitive equilibrium of $\&$. Then $\left(x^{*}, \theta^{*}\right)$ belongs to $\operatorname{SC}(\varepsilon)$.

Proof. Suppose for some $\bar{s} \in S$ there exists a coalition $C \in \mathcal{C}$ which can SC-improve upon $\left(x^{*}, \theta^{*}\right)$ by $\left(x^{C}, \theta^{C}\right) \in X^{C} \times \Theta^{C}$. Hence, we know that

$u^{h}\left(x^{h}\right)>u^{h}\left(x^{* h}\right), \quad h \in C$.

Because $\left(x^{* h}, \theta^{* h}\right)$ is a utility maximizing choice, $x_{s}^{h}=x_{s}^{* h}$ for all $s \neq \bar{s}, \theta^{h}=\theta^{* h}$, and (8) holds, one has for every $h \in C$,

$p_{\bar{S}}^{*} x_{\bar{s}}^{h}>p_{\bar{s}}^{*}\left(e_{\bar{s}}^{h}+D_{\bar{s}} \theta^{h}\right)$.

It follows from (9) and Definition 3.1.2 that

$$
\begin{aligned}
\sum_{h \in C} p_{\bar{s}}^{*}\left(e_{\bar{s}}^{h}+D_{\bar{s}} \theta^{h}\right) & <\sum_{h \in C} p_{\bar{s}}^{*} x_{\bar{s}}^{h}=p_{\bar{s}}^{*} \sum_{h \in C} x_{\bar{s}}^{h} \\
& =p_{\bar{s}}^{*} \sum_{h \in C}\left(e_{\bar{s}}^{h}+D_{\bar{s}} \theta^{h}\right),
\end{aligned}
$$

a contradiction.

Suppose that at date-event 0 there is a coalition $C \in \mathcal{C}$ which can SC-improve upon $\left(x^{*}, \theta^{*}\right)$ by $\left(x^{C}, \theta^{C}\right) \in X^{C} \times \Theta^{C}$, so

$u^{h}\left(x^{h}\right)>u^{h}\left(x^{* h}\right), \quad h \in C$.
Since $\left(x^{*}, \theta^{*}, p^{*}, q^{*}\right)$ is a competitive equilibrium, Theorem 6.2 implies that exists $\pi \in \mathbb{R}_{++}^{S}$ such that $q^{*}=\pi\left(p_{-0}^{*} \square A\right)$. By substituting all the budget constraints, one finds

$\sum_{s \in S^{\prime}} \pi_{s} p_{s}^{*} x_{s}^{* h}=\sum_{s \in S^{\prime}} \pi_{s} p_{s}^{*} e_{s}^{h}$

where we define $\pi_{0}=1$. We claim that for $h \in C, \sum_{s \in S^{\prime}} \pi_{s} p_{s}^{*} x_{s}^{h}>$ $\sum_{s \in S^{\prime}} \pi_{s} p_{s}^{*} e_{s}^{h}$. Suppose not, so for some $h \in C, \sum_{s \in S^{\prime}} \pi_{s} p_{s}^{*} x_{s}^{h} \leq$ $\sum_{s \in S^{\prime}} \pi_{s} p_{s}^{*} e_{s}^{h}$. Then, we have for $s \in S$,

$p_{s}^{*} x_{s}^{h}=p^{*} x_{s}^{* h}+p_{s}^{*} D_{s}\left(\theta^{h}-\theta^{* h}\right)=p_{s}^{*} e_{s}^{h}+p_{s}^{*} D_{s} \theta^{h}$,

and

$$
\begin{aligned}
p_{0}^{*} x_{0}^{h}+q^{*} \theta^{h} & =p_{0}^{*} x_{0}^{h}+\sum_{s \in S} \pi_{s} p_{s}^{*} D_{s} \theta^{h} \\
& =p_{0}^{*} x_{0}^{h}+\sum_{s \in S} \pi_{s} p_{s}^{*}\left(x_{s}^{h}-x_{s}^{* h}+D_{s} \theta^{* h}\right) \\
& \leq \sum_{s \in S^{\prime}} \pi_{s} p_{s}^{*} e_{s}^{h}-\sum_{s \in S} \pi_{s} p_{s}^{*} x_{s}^{* h}+p_{0}^{*} e_{0}^{h}-p_{0}^{*} x_{0}^{* h} \\
& =p_{0}^{*} e_{0}^{h}
\end{aligned}
$$

where we use Definition 3.1.2 for the second equality. It follows that $\left(x^{h}, \theta^{h}\right) \in B^{h}\left(p^{*}, q^{*}\right)$, which leads to a contradiction because $u^{h}\left(x^{h}\right)>u^{h}\left(x^{* h}\right)$. Consequently our claim holds true.

Applying this claim, Definitions 3.3.1 and 6.1, we have

$$
\begin{aligned}
\sum_{h \in C} \sum_{s \in S^{\prime}} \pi_{s} p_{s}^{*} x_{s}^{h} & =\sum_{h \in C} p_{0}^{*} x_{0}^{h}+\sum_{h \in C} \sum_{s \in S} \pi_{s} p_{s}^{*}\left(x_{s}^{* h}+D_{s}\left(\theta^{h}-\theta^{* h}\right)\right) \\
& =\sum_{h \in C} \sum_{s \in S^{\prime}} \pi_{s} p_{s}^{*} e_{s}^{h} \\
& <\sum_{h \in C} \sum_{s \in S^{\prime}} \pi_{s} p_{s}^{*} x_{s}^{h},
\end{aligned}
$$

a contradiction.

Hence, the competitive equilibrium $\left(x^{*}, \theta^{*}, p^{*}, q^{*}\right)$ belongs to the Segregated Core of the economy $\mathbb{E}$.

We show next that the competitive equilibrium belongs to the Two-stage Core as well.

Theorem 6.4. Let $\left(x^{*}, \theta^{*}, p^{*}, q^{*}\right)$ be a competitive equilibrium of $\varepsilon$. Then $\left(x^{*}, \theta^{*}\right)$ belongs to $\operatorname{TSC}(\mathcal{E})$.

Proof. Let $\left(x^{*}, \theta^{*}, p^{*}, q^{*}\right)$ be a competitive equilibrium. For dateevents $\bar{s} \in S$ there cannot exist a coalition $C \in \mathcal{C}$ which can TSC-improve upon $\left(x^{*}, \theta^{*}\right)$ by $\left(x^{C}, \theta^{C}\right) \in X^{C} \times \Theta^{C}$, since such an improvement would also be an SC-improvement, which is impossible by Theorem 6.3.

Suppose that at date-event 0 there is a coalition $C \in \mathcal{C}$ which can TSC-improve upon $\left(x^{*}, \theta^{*}\right)$ by $\left(x^{C}, \theta^{C}\right) \in X^{C} \times \Theta^{C}$, so

$u^{h}\left(x^{h}\right)>u^{h}\left(x^{* h}\right), \quad h \in C$.

Since $\left(x^{*}, \theta^{*}, p^{*}, q^{*}\right)$ is a competitive equilibrium, Theorem 6.2 implies that exists $\pi \in \mathbb{R}_{++}^{S}$ such that $q^{*}=\pi\left(p_{-0}^{*} \square A\right)$. By substituting all the budget constraints, one finds

$\sum_{s \in S^{\prime}} \pi_{s} p_{s}^{*} x_{s}^{* h}=\sum_{s \in S^{\prime}} \pi_{s} p_{s}^{*} e_{s}^{h}$ 


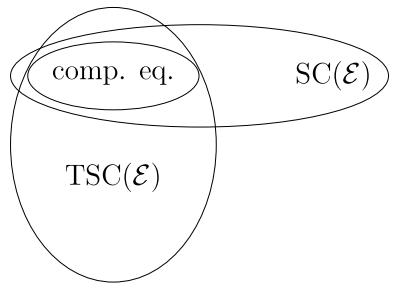

Fig. 5. The competitive equilibrium and the core.

where $\pi_{0}=1$ by definition. We claim that for $h \in C$,

$\sum_{s \in S^{\prime}} \pi_{s} p_{s}^{*} x_{s}^{h}>\sum_{s \in S^{\prime}} \pi_{s} p_{s}^{*} e_{s}^{h}$

Suppose not, so for some $h \in C, \sum_{s \in S^{\prime}} \pi_{s} p_{s}^{*} x_{s}^{h} \leq \sum_{s \in S^{\prime}} \pi_{s} p_{s}^{*} e_{s}^{h}$. Then, since for $s \in S$,

$p_{s}^{*} x_{s}^{h}=p_{s}^{*} e_{s}^{h}+p_{s}^{*} D_{s} \theta^{h}$

and

$$
\begin{aligned}
p_{0}^{*} x_{0}^{h}+q^{*} \theta^{h} & =p_{0}^{*} x_{0}^{h}+\sum_{s \in S} \pi_{s} p_{s}^{*} D_{s} \theta^{h} \\
& =p_{0}^{*} x_{0}^{h}+\sum_{s \in S} \pi_{s} p_{s}^{*} x_{s}^{h}-\sum_{s \in S} \pi_{s} p_{s}^{*} e_{s}^{h} \\
& \leq \sum_{s \in S^{\prime}} \pi_{s} p_{s}^{*} e_{s}^{h}-\sum_{s \in S} \pi_{s} p_{s}^{*} e_{s}^{h} \\
& =p_{0}^{*} e_{0}^{h},
\end{aligned}
$$

we find $\left(x^{h}, \theta^{h}\right) \in B^{h}\left(p^{*}, q^{*}\right)$, which leads to a contradiction because $u^{h}\left(x^{h}\right)>u^{h}\left(x^{* h}\right)$. Consequently our claim holds true.

Applying this claim, Definitions 3.4.1 and 6.1, we have

$$
\begin{aligned}
\sum_{h \in C} \sum_{s \in S^{\prime}} \pi_{s} p_{s}^{*} x_{s}^{h} & =\sum_{h \in C} p_{0}^{*} x_{0}^{h}+\sum_{h \in C} \sum_{s \in S} \pi_{s} p_{s}^{*}\left(e_{s}^{h}+D_{s} \theta^{h}\right) \\
& =\sum_{h \in C} \sum_{s \in S^{\prime}} \pi_{s} p_{s}^{*} e_{s}^{h} \\
& <\sum_{h \in C} \sum_{s \in S^{\prime}} \pi_{s} p_{s}^{*} x_{s}^{h},
\end{aligned}
$$

a contradiction.

Hence, the competitive equilibrium $\left(x^{*}, \theta^{*}, p^{*}, q^{*}\right)$ belongs to the Two-stage Core of the economy $\mathbb{E}$.

When markets are incomplete, a competitive equilibrium is typically not Pareto efficient. In fact, as in demonstrated in Geanakoplos and Polemarchakis (1986), Citanna et al. (1998), and Herings and Polemarchakis (2005) even constrained optimality concepts are typically not satisfied, although the heterogeneity of agents and the requirement of anonymity may interfere with improving interventions (Kajii, 1994). It follows that a competitive equilibrium in general does not belong to the Classical Core or to the Strong Sequential Core. Predtetchinski et al. (2006) present an example of an economy without assets markets and otherwise standard assumptions having an empty Weak Sequential Core. Since in such an economy competitive equilibria exist, it follows that also the Weak Sequential Core does in general not contain the competitive equilibria of an economy. Since competitive equilibria are not even constrained optimal, the fact that competitive equilibria may not belong to a dynamic core concept is a natural feature. In fact, that competitive equilibria always belong to the Segregated Core and the Two-stage Core is an indication that these concepts are too permissive.

The results of this section are summarized in Fig. 5.

\section{Multiple commodities and incomplete markets}

In this section we analyze the relationship of the core concepts (see Fig. 6) when there are multiple commodities and incomplete markets. We will argue, imposing Assumption 4.2.2, that $\operatorname{SSC}(\varepsilon) \subset$ WSC $(\mathscr{E}) \subset \mathrm{TSC}(\mathscr{E})$. Note that the first inclusion is an immediate consequence of Theorem 3.8.2.

Theorem 7.1. It holds under Assumption 4.2.2 that $\operatorname{WSC}(\mathscr{E}) \subset$ $\operatorname{TSC}(\&){ }^{4}$

Proof. Consider $(\bar{x}, \bar{\theta}) \in \operatorname{WSC}(\varepsilon)$. Let $C \in \mathcal{C}$ be a coalition that TSC-improves upon $(\bar{x}, \bar{\theta})$ by $\left(x^{C}, \theta^{C}\right) \in X^{C} \times \Theta^{C}$ at date-event 0 . We show that coalition $C$ can WSC-improve upon $(\bar{x}, \bar{\theta})$ at dateevent 0 by some $\left(\hat{x}^{C}, \theta^{C}\right)$, which leads to a contradiction since $(\bar{x}, \bar{\theta}) \in \operatorname{WSC}(\mathcal{E})$.

According to Definitions 3.4.1 and 3.4.2,

$x_{-0}^{h}=e_{-0}^{h}+A \theta^{h}, \quad h \in C$,

$\sum_{h \in C} x_{0}^{h}=\sum_{h \in C} e_{0}^{h}$

$\sum_{h \in C} \theta^{h}=0$.

We claim that $\operatorname{CC}\left(\varepsilon_{s, x^{C}, \theta^{C}}\right)$ is non-empty. Notice that $\bar{e}_{s, s}^{h}=e_{s}^{h}+$ $D_{s} \theta^{h}=x_{s}^{h}$ and $\bar{e}_{s,-s}^{h}=x_{-s}^{h}$, so $\bar{e}_{s}^{h}=x^{h} \in X^{h}$. Since $(\bar{x}, \bar{\theta}) \in \operatorname{WSC}(\varepsilon)$, it cannot be WSC-improved upon at date-event 0 by any coalition $\{h\}$, so $u^{h}\left(\bar{x}^{h}\right) \geq u^{h}\left(e^{h}\right), h \in H$. Since coalition C TSC-improves upon $(\bar{x}, \bar{\theta})$ at date-event 0 by $\left(x^{C}, \theta^{C}\right)$, we have $u^{h}\left(\bar{e}_{s}^{h}\right)=u^{h}\left(x^{h}\right)>$ $u^{h}\left(\bar{x}^{h}\right) \geq u^{h}\left(e^{h}\right), h \in C$. By Assumption 4.2.2, CC $\left(\varepsilon_{s, x^{C}, \theta^{C}}\right) \neq \emptyset$. For $s \in S, h \in C$, we choose $\hat{x}_{s}^{h}$ corresponding to an element in $\operatorname{CC}\left(\varepsilon_{s, x^{C}, \theta^{c}}\right)$ and we define $\hat{x}_{0}^{h}=x_{0}^{h}$. Our maintained assumption that utility functions are separable for states in $S$ implies that $\hat{x}^{C} \in$

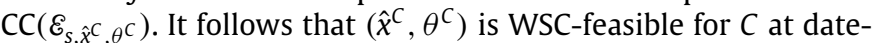
event 0 . Since

$u^{h}\left(\bar{x}^{h}\right)<u^{h}\left(x^{h}\right)=\sum_{s \in S} u_{s}^{h}\left(x_{0}^{h}, x_{s}^{h}\right) \leq \sum_{s \in S} u_{s}^{h}\left(\hat{x}_{0}^{h}, \hat{x}_{s}^{h}\right)=u^{h}\left(\hat{x}^{h}\right)$,

it is also a WSC-improvement.

Theorems 3.8.2 and 7.1 together yield that $\operatorname{SSC}(\varepsilon) \subset \operatorname{WSC}(\varepsilon) \subset$ $\operatorname{TSC}(\&)$. In general, the inclusions may be strict. The example of a finance economy without asset markets demonstrates that the first inclusion is typically strict. The results of Section 6 demonstrate that a competitive equilibrium belongs to the Two-stage Core but not necessarily to the Weak Sequential Core, so also the second inclusion is strict in general.

We know by Theorem 3.8.3 that $\operatorname{SSC}^{*}(\mathcal{E}) \subset \mathrm{CC}(\mathcal{E})$ and we have already argued in the setting of finance economies that there is no general relationship between the Classical Core on the one hand and the Weak Sequential Core, the Two-stage Core, and the Segregated Core on the other hand.

The questions that remain are the other relationships involving the Segregated Core. It has already been observed that the Segregated Core may contain elements that are not individually rational, so even the Two-stage Core is in general not a superset of the Segregated Core. Section 4 contains an example of an economy where the Segregated Core rules out allocations that belong to the Strong Sequential Core, but there, in the context of strongly complete markets, the example concerned an economy for which the weakly Pareto optimal allocations are distinct from the strongly Pareto optimal ones. In finance economies the Segregated Core coincides with the Two-stage

\footnotetext{
4 This result extends Observation 1 in Predtetchinski et al. (2006).
} 

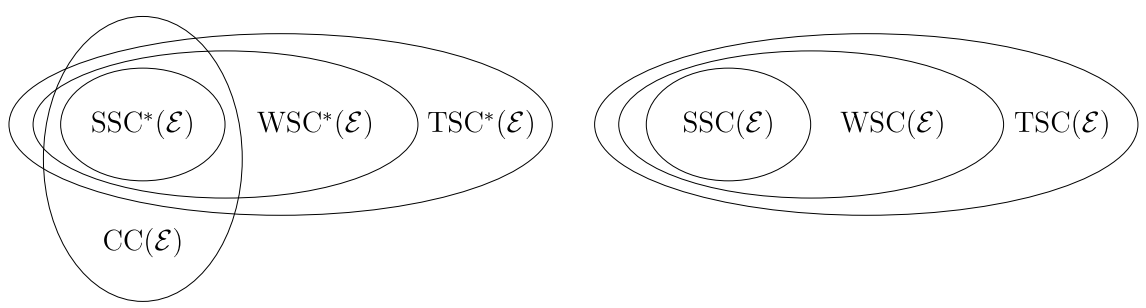

Fig. 6. Relationship of the core concepts-general case.

Core, even when markets are incomplete. We show now that in the multiple-commodity case, when markets are incomplete the Segregated Core may rule out allocations that belong to the Strong Sequential Core, even when all the assumptions of Section 4 are satisfied.

Example 7.2. Consider an economy $\varepsilon$ without uncertainty, two households, two commodities, and no asset markets, $S=\{1\}, H=$ $\{1,2\}, L=\{1,2\}$, and $J=\emptyset$. The households' initial endowments are

$\left(e_{0}^{1}, e_{0}^{2}\right)=\left(\begin{array}{ll}1 & 0 \\ 0 & 1\end{array}\right)$ and $\left(e_{1}^{1}, e_{1}^{2}\right)=\left(\begin{array}{ll}1 & 0 \\ 0 & 1\end{array}\right)$.

We define the consumption sets as $X^{1}=X^{2}=\mathbb{R}_{+}^{2} \times \mathbb{R}_{+}^{2}$. The utility functions are given by

$u^{1}\left(x^{1}\right)=x_{0,1}^{1} x_{1,1}^{1} x_{1,2}^{1}+x_{0,2}^{1} x_{1,1}^{1} x_{1,2}^{1}$,

$u^{2}\left(x^{2}\right)=x_{0,1}^{2} x_{1,1}^{2} x_{1,2}^{2}+x_{0,2}^{2} x_{1,1}^{2} x_{1,2}^{2}$.

Notice that this economy satisfies Assumptions 4.2.2 and 4.2.3. ${ }^{5}$

Consider the following allocation,

$\left(\bar{x}_{0}^{1}, \bar{x}_{0}^{2}\right)=\left(\begin{array}{cc}\frac{1}{4} & \frac{3}{4} \\ \frac{1}{4} & \frac{3}{4}\end{array}\right)$ and $\left(\bar{x}_{1}^{1}, \bar{x}_{1}^{2}\right)=\left(\begin{array}{cc}\frac{1}{4} & \frac{3}{4} \\ \frac{1}{4} & \frac{3}{4}\end{array}\right)$.

We claim that this allocation belongs to the Strong Sequential Core, but not to the Segregated Core.

1. $\bar{x} \in \operatorname{SSC}^{*}(\varepsilon)$.

None of the singleton coalitions can block the allocation $\bar{x}$, since the utility that can be achieved by such a coalition is 0 at every date-event. Since $\bar{x}$ is Pareto optimal it cannot be SSC-improved upon by coalition $\{1,2\}$ at any date-event.

2. $\bar{x} \notin \mathrm{SC}^{*}(\varepsilon)$.

The only SC-feasible allocation for coalition $\{1\}$ at date-event 0 is

$$
\left(x_{0}^{1}, x_{1}^{1}\right)=\left(\begin{array}{ll}
1 & \frac{1}{4} \\
& \frac{1}{4}
\end{array}\right) \text {. }
$$

Since $1 / 16=u^{1}\left(x^{1}\right)>u^{1}\left(\bar{x}^{1}\right)=1 / 32$, coalition $\{1\}$ has an SC-improvement at date-event 0 .

Summarizing the results of the section, we have that

$\operatorname{SSC}(\varepsilon) \subset \operatorname{WSC}(\varepsilon) \subset \operatorname{TSC}(\varepsilon)$.

\section{Conclusion}

In the literature a number of proposals can be found for the appropriate notion of the core in a context with restricted

\footnotetext{
5 The utility functions do not satisfy local non-satiation at date-events 0 and 1 , but this could easily be achieved by taking consumption sets such that zero consumption of a commodity in a date-event is excluded.
}

commitment possibilities. The environments studied are as distinct as economies with incomplete markets, economies with transaction costs, dynamic monetary economies, deterministic capital accumulation models, and sequences of transferable utility games. This paper unifies various treatments of dynamic core concepts that so far are scattered around in the literature, resulting in definitions of the Strong Sequential Core, the Weak Sequential Core, the Two-stage Core, and the Segregated Core in a common environment.

Our reformulation makes clear that the differences among the dynamic core concepts arise solely from the different requirements imposed on coalitions deviating at time-period 0. In the Segregated Core the net-trade is fixed. This implies, in contrast with all the other concepts, that the deviating coalition can in a sense use the endowments of non-coalition members in the following timeperiod. The Two-stage Core takes the completely conservative viewpoint that members of a deviating coalition cannot engage in any further trade in the following period; one just consumes the sum of the initial endowment and the payoff of the portfolio holdings one has agreed upon. Contrary to the previous concepts, the Strong Sequential Core allows for arbitrary trades inside the deviating coalition in each date-event. Thus the Strong Sequential Core is a refinement of the Classical Core for dynamic settings. The Weak Sequential Core allows only for those coalitional deviations, which are credible; there should not be a counter-deviation in the following period.

The need for the extension of the Classical Core is proved by the fact that even a complete set of assets is not sufficient for the equivalence of the resulting Classical Core and the dynamic concepts. A number of further assumptions need to be imposed to obtain this result. Also the Segregated Core is problematic as individual rationality is violated.

In the setting of finance economies the Classical Core turns out to be inappropriate again, and its outcomes are not related to the dynamic core ones. The Segregated Core, the Two-stage Core, and the Weak Sequential Core are proved to be equivalent in the one-commodity case, while blocking in the Strong Sequential Core is easier, and thus it is a subset of them. In this setting, the Strong Sequential Core is typically empty-valued, which makes it an unreasonable solution concept.

In general, the Strong Sequential Core is a subset of the Weak Sequential Core, which is a subset of the Two-stage Core and they are unrelated to the Segregated Core. The competitive equilibrium belongs to the Two-stage Core and to the Segregated Core but it may not belong to the other concepts. This property is perhaps less natural than it may seem as it is well-known that competitive equilibria are constrained suboptimal when asset markets are incomplete. It is therefore reasonable that this feature is recognized by an appropriate core concept; dynamic cooperation may overcome the inefficiencies of a competitive equilibrium in an incomplete markets setting. The Strong Sequential Core shares the weaknesses of the Classical Core, being a subset of it. Moreover, it is empty-valued for large classes of economies. All this leaves the Weak Sequential Core as the most satisfactory concept studied so far. 


\section{Acknowledgments}

The first author would like to thank the Hungarian Academy of Sciences for the financial support under the Momentum Programme (LD-004/2010) and OTKA-101106. The second author would like to thank the Netherlands Organisation for Scientific Research (NWO) for the financial support.

\section{References}

Becker, R.A., Chakrabarti, S.K., 1995. The recursive core. Econometrica 63 (2), 401-423.

Bester, H., 1984. Core and equilibrium in incomplete markets. Journal of Economics 44 (3), 255-266

Citanna, A., Kajii, A., Villanacci, A., 1998. Constrained suboptimality in incomplete markets: a general approach and two applications. Economic Theory 11 (3), 495-521.

Gale, D., 1978. The core of a monetary economy without trust. Journal of Economic Theory 19 (2), 456-491.

Geanakoplos, J., Polemarchakis, H.M., 1986. Existence, regularity, and constrained suboptimality of competitive allocations when the asset market is incomplete.
In: Heller, R.S.W., Starrett, D. (Eds.), Uncertainty, Information and Communication: Essays in Honor of K.J. Arrow, vol. III. Cambridge University Press, Cambridge, pp. 65-96.

Grossman, S.J., 1977. A characterization of the optimality of equilibrium in incomplete markets. Journal of Economic Theory 15 (1), 1-15.

Herings, P.J.J., Polemarchakis, H., 2005. Pareto improving price regulation when the asset market is incomplete. Economic Theory 25 (1), 135-154.

Kajii, A., 1994. Anonymity and optimality of competitive equilibria when markets are incomplete. Journal of Economic Theory 64 (1), 115-129.

Koutsougeras, L.C., 1998. A two-stage core with applications to asset market and differential information economies. Economic Theory 11 (3), 563-584.

Kranich, L., Perea, A., Peters, H., 2005. Core concepts for dynamic TU games. International Game Theory Review 7 (1), 43-61.

Predtetchinski, A., Herings, P.J.J., Perea, A., 2006. The weak sequential core for two-period economies. International Journal of Game Theory 34 (1), 55-65.

Predtetchinski, A., Herings, P.J.J., Peters, H., 2002. The strong sequential core for twoperiod economies. Journal of Mathematical Economics 38 (4), 465-482. (18).

Repullo, R., 1988a. The core of an economy with transaction costs. Review of Economic Studies 55 (3), 447-458.

Repullo, R., 1988b. A new characterization of the efficiency of equilibrium with incomplete markets. Journal of Economic Theory 44 (2), 217-230.

Roth, A.E., Postlewaite, A., 1977. Weak versus strong domination in a market with indivisible goods. Journal of Mathematical Economics 4 (2), 131-137. 